Imperial-TP-AT-2007-2

\title{
Strong-coupling expansion of cusp anomaly and gluon amplitudes from quantum open strings in $A d S_{5} \times S^{5}$
}

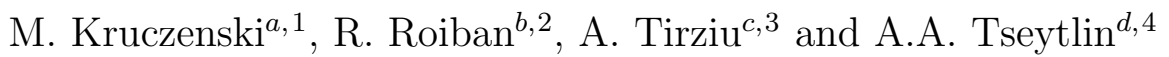 \\ ${ }^{a}$ Department of Physics, Purdue University, \\ W. Lafayette, IN 47907-2036, USA \\ ${ }^{b}$ Department of Physics, The Pennsylvania State University, \\ University Park, PA 16802, USA \\ ${ }^{c}$ Department of Physics, The Ohio State University, \\ Columbus, OH 43210, USA \\ ${ }^{d}$ Blackett Laboratory, Imperial College, London SW7 2AZ, U.K.
}

\begin{abstract}
An important "observable" of planar $\mathcal{N}=4$ SYM theory is the scaling function $f(\lambda)$ that appears in the anomalous dimension of large spin twist 2 operators and also in the cusp anomaly of light-like Wilson loop. The non-trivial relation between the anomalous dimension and the Wilson loop interpretations of $f(\lambda)$ is well-understood on the perturbative gauge theory side of the AdS/CFT duality. In the first part of this paper we present the dual string-theory counterpart of this relation, i.e. the equivalence between the closed-string and the open-string origins of $f(\lambda)$. We argue that the coefficient of the $\log S$ term in the energy of the closed string with large spin $S$ in $A d S_{5}$ should be equal to the coefficient in the logarithm of expectation value of the null cusp Wilson loop, to all orders in $\lambda^{-1 / 2}$ expansion. The reason is that the corresponding minimal surfaces happen to be related by a conformal transformation (and an analytic continuation). As a check, we explicitly compute the leading 1-loop string sigma model correction to the cusp Wilson loop, reproducing the same subleading coefficient in $f(\lambda)$ as found earlier in the spinning closed string case. The same function $f(\lambda)$ appears also in the resummed form of the 4-gluon amplitude as discussed at weak coupling by Bern, Dixon and Smirnov and recently found at the leading order at strong coupling by Alday and Maldacena (AM). Here we attempt to extend the latter approach to a subleading order in $\lambda^{-1 / 2}$ by computing the IR singular part of the 1-loop string correction to the corresponding T-dual Wilson loop. We discuss explicitly the 1-cusp case and comment on apparent problems with the dimensional regularization proposal of AM when directly applied order by order in strong coupling (string inverse tension) expansion.
\end{abstract}

\footnotetext{
${ }^{1}$ markru@purdue.edu

${ }^{2}$ radu@phys.psu.edu

3 tirziu@mps.ohio-state.edu

${ }^{4}$ Also at Lebedev Institute, Moscow. tseytlin@imperial.ac.uk
} 


\section{Introduction}

As is well known, in perturbative (planar $\mathcal{N}=4$ ) gauge theory there are two alternative routes that lead to the same scaling function $f(\lambda)$ : it can be found as a coefficient in the anomalous dimension of gauge-invariant large spin twist two operator [1, 2] or as a cusp anomaly of a light-like Wilson line [3, 4, 5, 6]. One can give a general proof of the equivalence between the two pictures in perturbative gauge theory [5].

On the dual perturbative $A d S_{5} \times S^{5}$ string theory side the anomalous dimension of minimal twist operator is represented by the energy of a closed string with large spin $S \gg 1$ in $A d S_{5}$ [7], $E=S+f(\lambda) \ln S+\ldots, \quad f(\lambda)_{\lambda \gg 1}=\frac{\sqrt{\lambda}}{\pi}+\ldots$. The same result for the strong-coupling limit of $f(\lambda)$ was shown in [8] (see also [9]) to follow from the open string picture, i.e. from the area of a surface ending on a cusp formed by two light-like Wilson lines on the boundary of $A d S_{5}$.

The definitions of $f(\lambda)$ in [7] and [8] seem very different and a priori unrelated, in contrast to the known perturbative gauge theory equivalence of the anomalous dimension of large spin twist 2 operator and the Wilson loop cusp anomaly function. In particular, while it was possible to compute the two subleading quantum corrections to $f(\lambda)$ in the closed spinning string picture [10, 11, 12$]$

$$
f(\lambda)_{\lambda \gg 1}=a_{0} \sqrt{\lambda}+a_{1}+\frac{a_{2}}{\sqrt{\lambda}}+\ldots, \quad a_{0}=\frac{1}{\pi}, \quad a_{1}=-\frac{3}{\pi} \ln 2,
$$

the direct computation of the quantum string corrections in the Wilson loop approach [13, 14, 8] appeared to be harder (for previous attempts in that direction see [15, 16, 17]).

One of our aims below will be to explain the relation between these two approaches, making their equivalence manifest (to all orders in the strong-coupling, i.e. $\frac{1}{\sqrt{\lambda}}$ expansion). In particular, we shall demonstrate that computing the quantum open $A d S_{5} \times S^{5}$ string fluctuations near the cusp surface of [8] leads to the same 1-loop coefficient $a_{1}$ in (1.1) as found in the closed-string picture in [10].

The key observation [10, 11] that simplifies dramatically the computation of quantum string corrections to the closed string energy in the large spin limit is that in order to compute the coefficient of the leading $\ln S$ term in $E$ it is enough to consider a "scaling" limit of the full (elliptic function) solution of [7]. In this limit the string is stretched homogeneously along the radial direction of $A d S_{5}$ all the way to the boundary, i.e. $\rho=\kappa \sigma$, with $\kappa \approx \frac{1}{\pi} \ln \frac{S}{\sqrt{\lambda}} \gg 1$. One may also ignore the boundary (turning-point) contributions since they are subleading in the large $\frac{S}{\sqrt{\lambda}}$ limit. This scaling-limit solution is formally related [11, 12] via an analytic continuation to the circular rotating string with two equal $S^{5}$ angular momenta $J_{1}=J_{2}$ [18, 19] and an imaginary value of the winding parameter. This reveals a simple "homogeneous" nature of this string background: the only non-trivial fields are isometric angles which are linear in the world-sheet coordinates $(\sigma, \tau)$, i.e. their derivatives are constant and so are the coefficients in the fluctuation Lagrangian. This makes the computation of the quantum corrections rather straightforward.

\footnotetext{
${ }^{1}$ The expression for $a_{2}$ can be found in [12].
} 
Remarkably, the cusp Wilson loop solution also admits [8] a similar simple "scaling" limit in which the Euclidean open-string world surface ends on two light-like lines on the boundary. This limit (combined with a regularization of the world-sheet area) was enough to reproduce [8] the leading term $a_{0}$ in the strong-coupling expansion (1.1) of the cusp anomaly function.

As we shall explain below in section 2, the two limiting ("scaling") solutions are actually closely related: they become equivalent upon certain analytic continuation (that is needed in particular to convert the Minkowski world sheet coordinates in the closed spinning string case into the Euclidean one in the open string Wilson loop case)2 2 and an $A d S_{5}$ isometry, i.e. a conformal $S O(2,4)$ transformation.

That relation implies that all quantum string corrections to the two partition functions which are computed by expanding the string action near the equivalent classical solutions should also agree. The logarithm of the string partition function in the conformal gauge determines (after dividing by the time interval) the quantum string corrections to the closed string energy in the scaling limit [11, 12]. The same open-string partition function on the disc leads (after an appropriate regularization of the world-sheet area [8]) to the cusp anomaly function. Given the general nature of the relation between the two scaling string solutions (and their homogeneous nature allowing one to ignore boundary effects) the equivalence of the quantum corrections to the corresponding string partition functions and thus to the $f(\lambda)$ function should thus extend to all orders in strong coupling expansion. This provides a proof of the equivalence between the two definitions of the scaling function also on the dual string-theory side, which is another remarkable manifestation of the AdS/CFT duality.

We will explicitly check this general statement in section 2.2 by first showing that the null cusp solution of [8] has a hidden "homogeneous" structure: the Lagrangian for string fluctuations near it has indeed (after an appropriate field redefinition) constant coefficients and produces the same characteristic spectrum as in the scaling limit of the spinning closed string solution in [10, 11]. This implies that the 1-loop correction to the null cusp Wilson loop leads to the same $a_{1}$ coefficient in $f(\lambda)$ in (1.1) as found in the spinning closed string picture.

The cusp anomalous dimension of the Wilson loop (which in gauge theory is determined by a UV singularity) governs also [20] the IR asymptotics of the gluon amplitudes in QCD and in $\mathcal{N}=4 \mathrm{SYM}$ theory. The on-shell gluon amplitudes are IR divergent; in dimensional regularization $D=4-2 \epsilon, \epsilon<0$ the IR poles in $\epsilon$ exponentiate and the double pole is determined in terms of the same $f(\lambda)$ function [22]. Moreover, $f(\lambda)$ controls also the finite $\ln ^{2} \frac{s}{t}$ part of the exponentiated form of the 4-gluon amplitude [23, 24] (which is apparently determined by conformal invariance considerations 3 relating it the IR singular part [25]).

The same exponential expression for the 4-gluon amplitude was found also at the leading order in strong coupling expansion using the AdS/CFT correspondence in [26]. Symbolically,

\footnotetext{
${ }^{2} \mathrm{~A}$ similar analytic continuation (in spin) was used to relate the anomalous dimension of twist 2 operators to the Wilson loop cusp anomaly picture on the gauge theory side [5] (which involved also separating the fields in the twist 2 operator and inserting a Wilson line).

${ }^{3}$ This is apparently no longer so for $n$-point amplitudes [40].
} 
assuming some IR cutoff $\mu \rightarrow 0$, the IR singular part of the 4-gluon amplitude may be written as

$$
\mathcal{A}_{4} \sim\left[\mathcal{A}_{d i v}(\mu, s) \mathcal{A}_{d i v}(\mu, t)\right]^{2}, \quad \mathcal{A}_{d i v}(\mu, s)=\exp \left[-\frac{1}{8} f(\lambda) \ln ^{2} \frac{\mu^{2}}{|s|}-\frac{1}{4} g(\lambda) \ln \frac{\mu^{2}}{|s|}\right],
$$

where $s=-\left(k_{1}+k_{2}\right)^{2}$ and $g(\lambda)$ is a non-universal function depending on a choice of the IR cutoff. As was found in [26] at the classical string order using a special "dual" version of the dimensional regularization prescription

$$
f(\lambda)=\frac{\sqrt{\lambda}}{\pi}+\mathcal{O}(1), \quad g(\lambda)=\frac{\sqrt{\lambda}}{2 \pi}(1-\ln 2)+\mathcal{O}(1) .
$$

Our aim in the second part of this paper (section 3) will be to attempt to extend the proposal of [26] to the quantum (1-loop) string level. One motivation is to explicitly check that it is the same universal scaling function $f(\lambda)$ that indeed appears in the exponential form of the 4-gluon amplitude when computed in strong coupling expansion. Another is to clarify the structure of the large $\lambda$ expansion of the second scaling function $g(\lambda)$ with a hope of understanding the interpolation to the 2-loop [23] and 3-loop weak-coupling result for it in [24].

The proposal of [26] was based on starting with a "dimensionally extended" analog of the near-horizon D3-brane background to which one should apply T-duality transformation along the $D=4-2 \epsilon$ longitudinal directions.

The use of the $2 \mathrm{~d}$ duality transformation or, in the target space language, of the T-duality transformation was one of the key observations of [26] that suggested a relation between the strong-coupling (semiclassical) computation of the 4-gluon amplitude and the computation of the null cusp Wilson loop. Thinking of gluons as represented by open strings (attached to D3-branes) and considering the leading strong-coupling approximation one may expect (by analogy with high-energy asymptotics of string scattering amplitudes in flat space [27]) that their scattering amplitude should be dominated by a semiclassical string solution depending on some fixed light-like 4-momenta $k_{i}^{m}$ (i.e. on the $s$ and $t$ kinematic variables in the 4-gluon case). At a qualitative level, since for open strings in flat space the T-duality exchanges the Neumann and Dirichlet boundary conditions [28], it should transform a configuration of strings with free ends (thus having specified conserved target-space momenta) to the one with the ends fixed at certain positions. As discussed in [26], in the T-dual picture the open string world surface should then end on a closed contour with straight sides determined by the light-like momenta $k_{i} \cdot 4$ The resulting world surface is then related (before an IR regularization) by an $S O(2,4)$

\footnotetext{
${ }^{4}$ Consider a flat target space and the open-string world sheet as a half-plane $(\tau>0,-\infty<\sigma<\infty)$. Then a string solution $x^{m} \sim k^{m} \ln \left(\sigma^{2}+\tau^{2}\right)$ that is sourced by an external momentum term (originating from the vertex operator insertions as in [27]) is a semi-infinite line representing a point-like string coming from infinity with momentum $k^{m}$. The formal dual solution $\left(\partial_{a} \tilde{x}^{m}=\epsilon_{a b} \partial_{b} x^{m}\right)$ is $\tilde{x}^{m} \sim k^{m} \arctan \frac{\tau}{\sigma}$ and near the origin it is a segment of length $\pi k^{m}$ instead of semi-infinite line. Formally, the $2 \mathrm{~d}$ duality is an equivalence in the absence of source terms, but the argument should still go through for sources that are localized, i.e. are of delta-function type.
} 
transformation [26] to the cusp Wilson loop surface found in [8] and discussed below in section 2 .

This T-duality transformation appears thus to relate the scattering amplitudes to the "momentum space" Wilson loops [29, 20, 25]. The latter were first discussed in a closely related gauge - string duality context in [30].

In general, given a string sigma model with the metric $d s^{2}=G(z) d x^{m} d x^{m}+d z^{2}+\ldots$ the corresponding classical solution can be related to a classical solutions in the T-dual metric $d \tilde{s}^{2}=G^{-1}(z) d y^{m} d y^{m}+d z^{2}+\ldots$ via $y^{m}=\tilde{x}^{m}, G(z) \partial_{a} x^{m}=\epsilon_{a b} \partial^{b} \tilde{x}^{m}$. The case of the standard $A d S_{5}$ in Poincare coordinates is special $\sqrt{6}$ since here the original $d s^{2}=z^{-2}\left(d x^{m} d x^{m}+d z^{2}\right)$ and the dual $d \tilde{s}^{2}=z^{2} d y^{m} d y^{m}+z^{-2} d z^{2}$ metrics are, in fact, related by the coordinate transformation (interchanging the boundary and the horizon): $z \rightarrow z^{-1}$. Here one may say that the 2-d duality acts on the same space of all classical solutions, provided we combine it with the coordinate transformation $z \rightarrow z^{-1}$. In particular, both the original scattering solution and its Wilson loop counterpart may be viewed, at the classical level, as solutions of the same $A d S_{5}$ sigma model, albeit with different boundary conditions. That does not mean that solutions related by such transformation are equivalent (given, in particular, that they satisfy different boundary conditions in $(\tau, \sigma)$ and thus describe different physical situations), but some of their properties are indeed closely related 7

In section 3.1 we shall first ignore the dimensional regularization aspect (imposing a formal regularization on the world sheet area only at the very end) and assume that the T-duality relation between the semiclassical world-sheet describing gluon scattering and the light-like cusped Wilson loop suggested in [26] extends beyond the classical level to the full quantum world-sheet theory as defined by the $A d S_{5} \times S^{5}$ superstring action [31]. The open string scattering solution in the original near-horizon D3-brane background, i.e. in $A d S_{5} \times S^{5}$ supported by the 5-form flux should thus be related to the light-like cusp Wilson loop surface in the T-dual background, i.e. in the near-core region of the smeared D-instanton solution. The latter has the same $A d S_{5} \times S^{5}$ metric but is supported by a dilaton and a RR 1-form background. As a result, while the bosonic sigma model part of the associated superstring action is the same, the fermionic part is formally different. A simple form of the corresponding superstring action was found in [15] by applying the $2 \mathrm{~d}$ duality to the Poincare patch $x^{m}$ coordinates in the action of [31] (written in a particular "Killing-spinor" $\kappa$-symmetry gauge). The transformation $x^{m} \rightarrow \tilde{x}^{m} \equiv y^{m}$ may be viewed as a quantum change of variables in the string partition function, so the two partition functions (when properly defined to account for the boundary conditions) should actually agree. Computing the 1-loop partition function in the T-dual geometry we shall indeed find the same result for the $a_{1}$ coefficient in $f(\lambda)$.

\footnotetext{
${ }^{5}$ There is an extra factor of $i$ in front of $\epsilon_{a b}$ in the case of the euclidean $2 \mathrm{~d}$ signature.

${ }^{6}$ Here we are ignoring a possible modification of $A d S_{5} \times S^{5}$ due to an IR regularization [26].

${ }^{7}$ Let us mention also that the above T-duality effectively inverting the $z$-coordinate inverts also the notion of the UV and IR singularities: the IR divergences of the amplitudes are mapped to UV divergences of the momentum-space Wilson loops (similar relation was already observed at weak coupling, see 25] and refs. there). Note also that in the dimensional regularization framework of 26 inverting $z$ effectively corresponds to changing sign of $\epsilon=\frac{4-D}{2}$.
} 
In section 3.2 we shall address the issue of how to extend the dimensional regularization prescription of [26] to the quantum string level. We will show how one can redo the 1-loop computation for the 1-cusp Wilson loop using the $D=4-2 \epsilon$ prescription of [26]. We will find that, contrary to what happened at the classical string level [26], keeping $\epsilon$ finite does not provide the required regularization of the result.

In section 3.3 we shall discuss several problems with the "quantum string" version of the dimensional regularization proposal of [26], suggesting that it may not apply order by order in the inverse string tension $\left(\frac{1}{\sqrt{\lambda}}\right)$ expansion.

The Wilson loop directly related to the 4-gluon scattering amplitude is the 4-cusp Wilson loop which, in the absence of the regularization, i.e. in $D=4$, can be found [26] by applying a conformal transformation to the 1-cusp [8] solution. In the regularized $D=4-2 \epsilon$ case finding this solution appears to be complicated (one can no longer use the conformal transformation trick). In Appendix A we describe the construction of the leading order $\epsilon$ term in this "regularized" solution and comment on the structure of the corresponding small fluctuation Lagrangian.

In appendix B we attempt to resolve the problems discussed in section 3.3 by suggesting a modification of the dimensional regularization prescription of [26] that may apply order by order in string perturbative expansion.

\section{Strong coupling expansion of the scaling function: equivalence of spinning closed string and null cusp Wilson loop pictures}

Below we shall use the following notation for coordinates in $A d S_{5}$ (we shall often set its radius to 1$)$. The global coordinates $\left(\rho, t, \phi, \theta_{1}, \theta_{2}\right)$

$$
d s^{2}=d \rho^{2}-\cosh ^{2} \rho d t^{2}+\sinh ^{2} \rho\left(d \phi^{2}+\cos ^{2} \phi d \theta_{1}^{2}+\sin ^{2} \phi d \theta_{2}^{2}\right)
$$

are related to the embedding coordinates $X_{M}(M=0, \ldots, 5)$ on which $S O(2,4)$ is acting linearly by

$$
\begin{gathered}
X_{0}+i X_{5}=\cosh \rho e^{i t}, \quad X_{1}+i X_{2}=\sinh \rho \cos \phi e^{i \theta_{1}}, \quad X_{3}+i X_{4}=\sinh \rho \sin \phi e^{i \theta_{2}}, \\
d s^{2}=d X^{M} d X_{M}, \quad X^{M} X_{M} \equiv-X_{0}^{2}-X_{5}^{2}+X_{1}^{2}+X_{2}^{2}+X_{3}^{2}+X_{4}^{2}=-1 .
\end{gathered}
$$

In the Poincare coordinates with the boundary at $z=0$ one has

$$
d s^{2}=\frac{1}{z^{2}}\left(d x^{m} d x_{m}+d z^{2}\right), \quad \quad x^{m} x_{m} \equiv-x_{0}^{2}+x_{i}^{2}, \quad i=1,2,3 .
$$

The relation to the embedding coordinates is

$$
X_{0}=\frac{x_{0}}{z}, \quad X_{i}=\frac{x_{i}}{z}, \quad X_{4}=\frac{1}{2 z}\left(-1+z^{2}+x^{m} x_{m}\right), \quad X_{5}=\frac{1}{2 z}\left(1+z^{2}+x^{m} x_{m}\right) .
$$




\subsection{Scaling limits of the spinning closed string and cusp Wilson loop minimal surfaces and their equivalence}

Let us start with recalling the scaling limit [10, 11] of the spinning closed string solution of [7]. Here we shall use the conformal gauge with Minkowski $2 \mathrm{~d}$ signature, $d s^{2}=-d \tau^{2}+d \sigma^{2}$. In the limit of large spin the spinning closed string [7] written in global coordinates (2.1) can be approximated by

$$
t=\kappa \tau, \quad \rho=\kappa \sigma, \quad \theta_{1}=\kappa \tau, \quad \phi=\theta_{2}=0, \quad \kappa \approx \frac{1}{\pi} \ln \frac{S}{\sqrt{\lambda}} \gg 1 .
$$

Here $\theta_{1}$ is the rotation angle and the string is folded and stretched along $\rho$ with $\rho_{*}= \pm \kappa \pi$ as turning points. Rescaling the world-sheet coordinates by $\kappa \rightarrow \infty$ one effectively decompactifies $\sigma$, i.e. one may consider the world sheet as a plane. The world-sheet area then scales as $\kappa^{2}$ and the classical space-time energy scales as $\kappa$, i.e. as $\ln S$. The same is true to all orders in quantum $\alpha^{\prime} \sim \frac{1}{\sqrt{\lambda}}$ expansion since the solution happens to be homogeneous: the fluctuation Lagrangian has constant coefficients. Thus the quantum fluctuation problem is translationally invariant and the quantum effective action or $\ln Z$ is proportional to the area $\sim \kappa^{2}$ to all orders in $\frac{1}{\sqrt{\lambda}}[12$.

Writing (2.6) in the embedding coordinates we get (after rescaling $\tau, \sigma$ by $\kappa$ and thus assuming that they take values in the infinite interval in the limit $\kappa \rightarrow \infty)$

$$
\begin{aligned}
& X_{0}=\cosh \sigma \cos \tau, \quad X_{5}=\cosh \sigma \sin \tau, \\
& X_{1}=\sinh \sigma \cos \tau, \quad X_{2}=\sinh \sigma \sin \tau, \quad X_{3}=X_{4}=0 \text {, } \\
& X_{0} X_{2}=X_{5} X_{1} \text {. }
\end{aligned}
$$

The solution (2.7) thus belongs to the same class of homogeneous string solutions as the rigid circular string found in [18, 19]. In fact, it is formally equivalent, upon an analytic continuation and re-interpretation of the parameters, to the background representing the circular rotating string in $S^{5}$ with two equal angular momenta [11].

Let us now find a counterpart of this solution with euclidean $2 \mathrm{~d}$ world sheet. If we set $\tau \rightarrow-i \tau$ then $X_{5}$ and $X_{2}$ in (2.7) become imaginary, effectively exchanging places in (2.3) while still preserving the $(--++++)$ signature of the $6 \mathrm{~d}$ metric of the embedding space. Then we can get a real $A d S_{5}$ string solution with euclidean world sheet by simply renaming the coordinates:

$$
X_{0}^{\prime}=X_{0}, \quad X_{1}^{\prime}=X_{1}, \quad X_{5}^{\prime}=-i X_{2}, \quad X_{2}^{\prime}=-i X_{5}, \quad X_{3}^{\prime}=X_{3}, \quad X_{4}^{\prime}=X_{4},
$$

where $-X_{0}^{\prime 2}-X_{5}^{\prime 2}+X_{1}^{\prime 2}+X_{2}^{\prime 2}+X_{3}^{\prime 2}+X_{4}^{\prime 2}=-1$, i.e.

$$
\begin{aligned}
& X_{0}^{\prime}=\cosh \sigma \cosh \tau, \quad X_{5}^{\prime}=\sinh \sigma \sinh \tau, \\
& X_{1}^{\prime}=\sinh \sigma \cosh \tau, \quad X_{2}^{\prime}=\cosh \sigma \sinh \tau, \quad X_{3}^{\prime}=X_{4}^{\prime}=0, \\
& X_{0}^{\prime} X_{5}^{\prime}=X_{1}^{\prime} X_{2}^{\prime} .
\end{aligned}
$$


As we shall see below, this euclidean world sheet counterpart of the scaling limit of the spinning closed string solution is directly related to the null cusp Wilson line solution of [8]. Note that written in the Poincare coordinates (2.5) the solution (2.10) becomes

$$
\begin{aligned}
z^{\prime} & =(\sinh \tau \sinh \sigma)^{-1}, \quad x_{0}^{\prime}=\operatorname{coth} \tau \operatorname{coth} \sigma, \\
x_{1}^{\prime} & =\operatorname{coth} \tau, \quad x_{2}^{\prime}=\operatorname{coth} \sigma, \quad x_{3}=0 .
\end{aligned}
$$

Next, let us review the cusp solution found in [8] in the Poincare coordinates (2.4), i.e.

$$
d s^{2}=\frac{1}{z^{2}}\left(d z^{2}-d u^{2}+u^{2} d \xi^{2}+d x_{2}^{2}+d x_{3}^{2}\right), \quad x_{0}=u \cosh \xi, \quad x_{1}=u \sinh \xi .
$$

The limiting minimal surface ending on two light-like lines at the boundary $z=0$ found in $[8]$ in the static gauge (i.e. with $(u, \xi)$ as world-sheet directions) is

$$
z=\sqrt{2} u=\sqrt{2\left(x_{0}^{2}-x_{1}^{2}\right)}, \quad x_{2}=x_{3}=0 .
$$

The corresponding induced $2 \mathrm{~d}$ metric has euclidean signature: $d s^{2}=\frac{1}{2}\left(\frac{d u^{2}}{u^{2}}+d \xi^{2}\right)$. To regularize the area of this surface one may assume that $\ell<u<L,-\frac{\gamma}{2}<\xi<\frac{\gamma}{2}$ where $L, \gamma \rightarrow \infty, \ell \rightarrow 0$ [8]. Then the coefficient in the area representing the strong-coupling limit of the cusp anomaly reproduces [8] the same value of the leading strong-coupling coefficient $a_{0}$ in the scaling function (2.6) (see also [9]).

The same solution in the conformal gauge $\left(d s^{2}=d \tau^{2}+d \sigma^{2}\right)$ is

$$
z=\sqrt{2} u, \quad u=e^{\sqrt{2} \tau}, \quad \xi=\sqrt{2} \sigma .
$$

Looking for a more general solution in the conformal gauge that satisfies the limiting $z=\sqrt{2} u$ condition one finds that it is the same as (2.15) up to an $S O(2)$ rotation in the $(\tau, \sigma)$ plane: 8

$$
\begin{gathered}
z=\sqrt{2} u, \quad u=e^{\alpha \tau-\beta \sigma}, \quad \xi=\alpha \sigma+\beta \tau, \quad \alpha^{2}+\beta^{2}=2, \\
x_{0}=e^{\alpha \tau-\beta \sigma} \cosh (\alpha \sigma+\beta \tau), \quad x_{1}=e^{\alpha \tau-\beta \sigma} \sinh (\alpha \sigma+\beta \tau), \quad x_{2}=x_{3}=0 .
\end{gathered}
$$

The two natural simple choices are $\alpha=\sqrt{2}, \beta=0$ in (2.15) and $\alpha=\beta=1$ that we will use below.

Using (2.5) the conformal-gauge solution can be written in embedding coordinates as

$$
\begin{array}{ll}
X_{0}=\frac{1}{\sqrt{2}} \cosh (\alpha \sigma+\beta \tau), & X_{5}=\frac{1}{\sqrt{2}} \cosh (\alpha \tau-\beta \sigma), \\
X_{1}=\frac{1}{\sqrt{2}} \sinh (\alpha \sigma+\beta \tau), & X_{4}=\frac{1}{\sqrt{2}} \sinh (\alpha \tau-\beta \sigma), \quad X_{2}=X_{3}=0 .
\end{array}
$$

\footnotetext{
${ }^{8}$ One may also consider a general $2 \mathrm{~d}$ conformal transformation on $(\tau, \sigma)$; the resulting value of the classical string action (area) will be formally the same before one introduces a regularization.
} 
We can get an equivalent form of this solution

$$
\begin{array}{ll}
X_{0}=\frac{1}{\sqrt{2}} \cosh (\alpha \sigma+\beta \tau), \quad X_{5}=\frac{1}{\sqrt{2}} \cosh (\alpha \tau-\beta \sigma), \\
X_{1}=\frac{1}{\sqrt{2}} \sinh (\alpha \sigma+\beta \tau), \quad X_{2}=\frac{1}{\sqrt{2}} \sinh (\alpha \tau-\beta \sigma), \quad X_{3}=X_{4}=0
\end{array}
$$

by performing a discrete $S O(2,4)$ transformation that interchanges $X_{2}$ and $X_{4}$,

$$
X_{2} \rightarrow X_{4}, \quad X_{4} \rightarrow X_{2} .
$$

Note that for the solution (2.19) 8 ]

$$
X_{0}^{2}-X_{1}^{2}=X_{5}^{2}-X_{2}^{2}=\frac{1}{2}
$$

The transformation (2.20) effectively produces a non-zero value of $x_{2}$ : if we "project" (2.19) back to the Poincare patch using (2.5) we get instead of (2.17)

$$
\begin{aligned}
& z=\sqrt{2} e^{\alpha \tau-\beta \sigma}, \quad x_{0}=e^{\alpha \tau-\beta \sigma} \cosh (\alpha \sigma+\beta \tau) \\
& x_{1}=e^{\alpha \tau-\beta \sigma} \sinh (\alpha \sigma+\beta \tau), \quad x_{2}=e^{\alpha \tau-\beta \sigma} \sinh (\alpha \tau-\beta \sigma), \quad x_{3}=0
\end{aligned}
$$

It is easy to check that (2.19) (or (2.18) ) satisfies the string equations and the conformal gauge constraints (in euclidean $2 \mathrm{~d}$ metric) written in the embedding coordinates:

$$
\begin{aligned}
& \partial^{a} \partial_{a} X_{M}-\Lambda X_{M}=0, \quad X^{M} X_{M}=-1, \quad \Lambda=\partial^{a} X^{M} \partial_{a} X_{M}=2, \\
& \partial_{\tau} X^{M} \partial_{\tau} X_{M}-\partial_{\sigma} X^{M} \partial_{\sigma} X_{M}=0, \quad \partial_{\tau} X^{M} \partial_{\sigma} X_{M}=0 .
\end{aligned}
$$

This is thus a special case of the constant Lagrange multiplier $(\Lambda=$ const) solutions for which $X_{M}$ satisfies a linear constant-mass $2 \mathrm{~d}$ equation discussed in [19]. Such string solutions include rigid circular rotating strings and are effectively "homogeneous". 9

Applying another discrete $S O(2,4)$ transformation one can show explicitly that (2.19) is a homogeneous solution, i.e. it can be put into the form where only the isometric angles of the $A d S_{5}$ metric (i.e. the analogs of $t, \theta_{1}, \theta_{2}$ in the parametrization (2.1)) are non-zero and linear in $(\tau, \sigma)$. As a result, the fluctuation Lagrangian will have constant coefficients (after an appropriate choice of the basis of fluctuation fields). To see this explicitly let us group $X_{M}$ as $\left(X_{0}^{2}-X_{1}^{2}\right)+\left(X_{5}^{2}-X_{2}^{2}\right)-\left(X_{3}^{2}+X_{4}^{2}\right)=1$ and introduce new global $A d S_{5}$ coordinates as

$$
X_{0} \pm X_{1}=r_{1} e^{ \pm p}, \quad X_{5} \pm X_{2}=r_{2} e^{ \pm q}, \quad X_{3} \pm i X_{4}=r_{3} e^{ \pm i h}
$$

where $r_{i}$ satisfy $r_{1}^{2}+r_{2}^{2}-r_{3}^{2}=1$, i.e.

$$
r_{1}=\cosh r \cos f, \quad r_{2}=\cosh r \sin f, \quad r_{3}=\sinh r .
$$

\footnotetext{
${ }^{9}$ Note that (2.18) cannot describe a regular closed string since it is not periodic in $\sigma$ (unless one considers a scaling limit as discussed above in which $\sigma$ is rescaled by a large parameter) but it may be interpreted as an open-string solution with an infinite range of $\sigma$.
} 
The $A d S_{5}$ metric written in terms of the independent coordinates $p, q, \gamma, r, f$ becomes:

$$
d s^{2}=-\cosh ^{2} r d f^{2}+d r^{2}+\cosh ^{2} r\left(\cos ^{2} f d p^{2}+\sin ^{2} f d q^{2}\right)+\sinh ^{2} r d h^{2} .
$$

Our solution is then

$$
r=0, \quad f=\frac{\pi}{4}, \quad p=\alpha \sigma+\beta \tau, \quad q=\alpha \tau-\beta \sigma, \quad h=0,
$$

i.e. is homogeneous 10

Finally, let us now demonstrate that the solution (2.19) is, in fact, $S O(2,4)$-equivalent to the euclidean world sheet version of the scaling limit of the spinning closed string solution in (2.10). Let us choose $\alpha=\beta=1$ in (2.19) and apply two discrete $S O(2)$ rotations in the $\left(X_{0}, X_{5}\right)$, and $\left(X_{1}, X_{2}\right)$ planes (which of course preserve the $(--++++)$ metric)

$$
\begin{array}{ll}
X_{0}^{\prime}=\frac{1}{\sqrt{2}}\left(X_{0}+X_{5}\right), & X_{5}^{\prime}=\frac{1}{\sqrt{2}}\left(X_{0}-X_{5}\right), \\
X_{1}^{\prime}=\frac{1}{\sqrt{2}}\left(X_{1}+X_{2}\right), & X_{2}^{\prime}=\frac{1}{\sqrt{2}}\left(X_{1}-X_{2}\right) .
\end{array}
$$

The resulting background is then exactly the same as in $(2.10),(2.11)$.

As was implicit in [8] and recently discussed in detail in [26] the Poincare-patch solution (2.15) interpreted in the global coordinates (2.19), (2.21) describes a surface ending on a closed line with four and not just one null cusp. The presence of the four cusps was made clear in [26] by applying the transformation (2.20) and the same $S O(2,4)$ transformation as in (2.29). The resulting Poincare patch solution is similar to the one in (2.12) (with $X_{0} \leftrightarrow X_{5}$, i.e. $\sinh \leftrightarrow \cosh , \operatorname{coth} \leftrightarrow \tanh )$ and it ends on a rectangular contour with 4 null cusps 11 Taking into account the euclidean continuation and the subsequent rotation in the $(\tau, \sigma)$ plane (going from $\alpha=\sqrt{2}, \beta=0$ case in (2.15) to the $\alpha=\beta=1$ in $(2.19),(2.29)$ ) one may relate the origin of the 4 cusps to the presence of the 4 special points in the spinning closed (and folded) string surface: the two ends where the $\sigma$-derivatives vanish and the center where the $\tau$-derivatives vanish 12

To conclude, we have seen that the scaling (large spin) limit [10, 11] of the spinning closed string solution of [7] is formally equivalent, upon an analytic continuation to the euclidean world sheet combined with a discrete $S O(2,4)$ rotation in $A d S_{5}$, to the global $A d S_{5}$ version of

\footnotetext{
${ }^{10}$ The metric (2.27) is of course related to $S^{5}$ metric by an analytic continuation so there is formally a similar homogeneous $S^{5}$ solution.

${ }^{11}$ The corresponding open-string solution ending on a rectangular contour at the boundary $z=0$ was interpreted in 26] as being 2d-dual ("T-dual") to the semiclassical solution describing the massless open string (gluon) 4-point scattering amplitude with the Mandelstam variables $s=t$.

${ }^{12}$ One may also think of the closed string as a combination of two coinciding open strings with the ends at the two folds; this also suggests the presence of 4 cusps in the joint (euclidean) world sheet surface.
} 
the null cusp solution of [8]. This explains the agreement between the leading strong-coupling expressions for the scaling (cusp anomaly) function found respectively in [7] and in [8].

This equivalence, combined with the homogeneous nature of the string background, implies that the correspondence between the two pictures - the spinning closed string energy (or the minimal twist anomalous dimension) and the cusp Wilson loop - extends also to the quantum string level. The homogeneity of the scaling-limit string solution implying the $(\tau, \sigma)$ translational invariance of the quantum fluctuation Lagrangian means that one can essentially ignore the difference in the boundary conditions in the closed and open string cases. The contributions of the quantum string fluctuations to the energy of the closed string or to the open string partition function will be equivalent since in this scaling limit they are the same at each interior point of the string world sheet, i.e. they are proportional to the (regularized) area of the world sheet.

To check this argument, below in section 2.2 we shall explicitly find the spectrum of quadratic fluctuations near the cusp open string surface (2.19) and verify that they indeed lead to the same 1-loop cusp anomaly coefficient $a_{1}$ in (1.1) as found [10] in the spinning closed string case. Incidentally, that apparently will be the first explicit computation of the 1-loop string correction to a (non-BPS) Wilson loop surface done so far in the literature.

Before turning to the discussion of the quantum fluctuations let us mention several possible generalizations of the above discussion. One immediate extension is to consider a more general cusp Wilson loop to include an angular momentum $J$ in $S^{5}$, in direct analogy with what was done in [10, 11] for the rotating string (large finite $J$ corresponds to operators with large finite twist). 13 It is of interest also to consider the generalization of the spinning closed string to spinning string with $n>2$ spikes [33] to find its scaling (large spin) limit and to try to identify then a related euclidean open string world surface 14

\subsection{1-loop string correction to null cusp Wilson loop expectation value}

Let us now go back to the conformal-gauge solution (2.15) or (2.19) with $\alpha=\sqrt{2}, \beta=0$ and compute the spectrum of small fluctuations near it, demonstrating explicitly that it is the same

${ }^{13}$ The scaling limit [11] of the $(S, J)$ folded closed string solution of [10] is the following generalization of (2.6): $t=\kappa \tau, \quad \rho=\sqrt{\kappa^{2}-\nu^{2}} \sigma, \quad \theta_{1}=\kappa \tau, \quad \varphi=\nu \tau$ where $\varphi$ is from $S^{5}$ and $J=\sqrt{\lambda} \nu$. As one can easily verify, the corresponding generalization of the cusp Wilson line solution in conformal gauge (2.15) corresponding to the metric (2.13) with an extra $d \varphi^{2}$ term and having euclidean-signature world-sheet metric is: $z=a u, u=$ $e^{b \tau}, \quad \xi=b \sigma, \varphi=\nu^{\prime} \tau$, where $a=\frac{\sqrt{2} b}{\sqrt{b^{2}+\nu^{\prime 2}}}$. The induced metric is then $d s^{2}=\frac{b^{2}}{2}\left(d \tau^{2}+d \sigma^{2}\right)$ so one is to set $b=\sqrt{2}$ to put it into the canonical form as we did in (2.15) (then $a=\frac{2}{\sqrt{2+\nu^{\prime 2}}}$ ). The parameter $\nu^{\prime}$ is related to $\nu$ in the closed string case by $\nu^{\prime}=i \frac{\nu}{\kappa}$ (in going from Minkowski world sheet solution for a closed string to euclidean world sheet solution for the open string in section 2.1 we rotated $\tau \rightarrow-i \tau$; we also rescaled $\tau$ and $\sigma$ by $\kappa$ ). Other Wilson loop solutions with rotation in $S^{5}$ were considered in 32 .

${ }^{14}$ Below we shall also discuss a generalization of the above cusp solution to the dimensionally regularized case, following the suggestion of [26. It would be interesting to find explicitly the corresponding form of the spinning closed string solution (dimensional continuation to $D=4-2 \epsilon$ breaks $S O(2,4)$ symmetry so this is non-trivial). In contrast to the area of the cusp the energy of the closed string should be regular in the $\epsilon \rightarrow 0$ limit. 
as in the case of the scaling limit of the spinning closed string in [10, 11]. That (together with homogeneity of the solution) will imply that the 1-loop correction to the cusp anomaly is the same as the 1-loop correction to the coefficient of $\ln S$ in the fast-spinning string energy.

Let us start with bosonic fluctuations. It is easiest to consider the string action in terms of the embedding coordinates, though one can get the same fluctuation spectrum using the Poincare coordinates (that will require a non-trivial choice of the quantum fluctuation fields see appendix A). The euclidean world-sheet Lagrangian in the conformal gauge is

$$
L=\frac{1}{2} \partial_{a} X^{M} \partial^{a} X_{M}+\frac{1}{2} \Lambda\left(X^{M} X_{M}+1\right),
$$

with the solution (2.19) satisfying (2.23). Introducing the fluctuations

$$
X_{M} \rightarrow X_{M}+\tilde{X}_{M}, \quad \Lambda \rightarrow \Lambda+\tilde{\Lambda}
$$

we obtain the quadratic part of the fluctuation Lagrangian with $\tilde{X}_{M}$ subject to a linear constraint $(\Lambda=2)$

$$
\tilde{L}_{2}=\frac{1}{2} \partial_{a} \tilde{X}^{M} \partial^{a} \tilde{X}_{M}+\tilde{X}^{M} \tilde{X}_{M}, \quad X^{M} \tilde{X}_{M}=0 .
$$

The explicit form of the latter constraint is

$$
\tilde{X}_{0} \cosh \sqrt{2} \sigma-\tilde{X}_{1} \sinh \sqrt{2} \sigma+\tilde{X}_{5} \cosh \sqrt{2} \tau-\tilde{X}_{2} \sinh \sqrt{2} \tau=0 .
$$

Performing the field redefinition $\left(\tilde{X}_{0}, \tilde{X}_{1}\right) \rightarrow\left(Z_{0}, Z_{1}\right)$

$$
Z_{0}=\tilde{X}_{0} \cosh \sqrt{2} \sigma-\tilde{X}_{1} \sinh \sqrt{2} \sigma, \quad Z_{1}=-\tilde{X}_{0} \sinh \sqrt{2} \sigma+\tilde{X}_{1} \cosh \sqrt{2} \sigma
$$

and similarly $\left(\tilde{X}_{5}, \tilde{X}_{2}\right) \rightarrow\left(Z_{5}, Z_{2}\right)$, the constraint (2.33) takes the form

$$
Z_{0}+Z_{5}=0
$$

This allows us to eliminate $Z_{5}$ from the fluctuation Lagrangian which then becomes

$$
\begin{aligned}
\tilde{L}_{2}= & -\partial^{a} Z_{0} \partial_{a} Z_{0}+\frac{1}{2}\left(\partial^{a} Z_{1} \partial_{a} Z_{1}+\partial^{a} Z_{2} \partial_{a} Z_{2}\right)-2 \sqrt{2}\left(Z_{1} \partial_{\sigma} Z_{0}-Z_{2} \partial_{\tau} Z_{0}\right) \\
& +\frac{1}{2}\left(\partial^{a} \tilde{X}_{3} \partial_{a} \tilde{X}_{3}+\partial^{a} \tilde{X}_{4} \partial_{a} \tilde{X}_{4}+2 \tilde{X}_{3}^{2}+2 \tilde{X}_{4}^{2}\right) .
\end{aligned}
$$

This fluctuation Lagrangian is essentially equivalent to the euclidean version of the one found in [10, 11] for the scaling limit of the spinning closed string solution. Diagonalizing it we get two massless modes (whose contribution is compensated by that of the two massless conformal gauge ghosts), one mode with mass 2 and two modes with mass $\sqrt{2}$. In addition, there are five massless modes from the fluctuations in $S^{5}$ directions.

The quadratic fermionic action [31] is given by 15

$$
L_{F 2}=i\left(\eta^{a b} \delta^{I J}-\epsilon^{a b}{ }_{S}^{I J}\right) \bar{\theta}^{I} \phi_{a}\left[\delta^{J K} \mathcal{D}_{b}-\frac{i}{2} \epsilon^{J K} \Gamma_{*} \phi_{b}\right] \theta^{K}
$$

\footnotetext{
${ }^{15}$ For details and notation see, e.g., [12]. In particular, here $M=0,1, \ldots, 9, \mathrm{~s}^{I J}=(1,-1), \Gamma_{*}=i \Gamma_{01234}$, etc.
} 


$$
\phi_{a}=e_{M}^{A} \partial_{a} x^{M} \Gamma_{A}, \quad \mathcal{D}_{a}=\partial_{a}+\frac{1}{4} \omega_{M}^{A B} \partial_{a} x^{M} \Gamma_{A B}
$$

It simplifies in the $\theta^{1}=\theta^{2} \kappa$-symmetry gauge used also in [10, 12, It is most straightforward to find the fermionic spectrum (after a continuation to euclidean world sheet) in the coordinates (2.27) in which the solution takes the explicitly homogeneous form (2.28) and thus all the coefficients in (2.37) are constant. As in [10], we find eight fermionic modes with mass 1.

As a result, the logarithm of the 1-loop euclidean partition function is (here we assume that the rescaled $\tau$ and $\sigma$ coordinates change in the infinite limits)

$$
\begin{gathered}
\Gamma_{1}=-\ln Z_{1}=V_{2} \int \frac{d^{2} p}{(2 \pi)^{2}} \mathcal{Z}_{1}\left(p^{2}\right) \\
\mathcal{Z}_{1}\left(p^{2}\right)=\frac{1}{2}\left[\ln \left(p^{2}+4\right)+2 \ln \left(p^{2}+2\right)+5 \ln p^{2}-8 \ln \left(p^{2}+1\right)\right] .
\end{gathered}
$$

Here the volume $V_{2}$ factorizes since our background is translationally invariant. Note that $V_{2}=\int_{-\infty}^{\infty} d \tau d \sigma=2 A_{2}$, where $A_{2}$ corresponds to an area of a single cusp (i.e. $V_{2}$ is twice the area of a half-plane world sheet as appropriate for the open string case).

Computing the integral one finds as in [10, 11, 12]

$$
\Gamma_{1}=\frac{V_{2}}{4 \pi} \int_{0}^{\infty} d v \mathcal{Z}_{1}(v)=a_{1} A_{2}, \quad a_{1}=-\frac{3 \ln 2}{\pi}
$$

For comparison, the value of the classical action is $\left(\sqrt{\lambda}=\frac{R^{2}}{\alpha^{\prime}}\right)$

$$
I=\frac{\sqrt{\lambda}}{2 \pi} \int_{-\infty}^{\infty} d \tau d \sigma \frac{1}{2} \partial^{a} X^{M} \partial_{a} X_{M}=\frac{\sqrt{\lambda}}{2 \pi} V_{2}=\sqrt{\lambda} a_{0} A_{2}, \quad a_{0}=\frac{1}{\pi} .
$$

The area of the cusp open-string world sheet regularized as in [8] is $A_{2}=\frac{\gamma}{4} \ln \frac{L}{\ell}$, where $\gamma \rightarrow \infty$ is a boost parameter for the two lines forming a cusp and $L$ and $\ell$ are the IR and the UV cutoffs 16

\section{Strong-coupling corrections to IR singular part of gluon scattering amplitude: dual Wilson loop expectation value}

In section 2.2 we have computed the 1-loop string correction to the 1-cusp Wilson loop and found that it contains the same 1-loop corrected $f(\lambda)$ function as appearing in the energy of the spinning closed string (and we argued that this agreement should hold also to all orders in strong coupling expansion). We used the standard $A d S_{5} \times S^{5}$ superstring action with the 5 -form coupling in the fermionic part.

\footnotetext{
${ }^{16}$ Imposing a cutoff at $z=\varepsilon \rightarrow 0$ in (2.13) implies that $\gamma_{\max } \sim \ln \frac{L}{\varepsilon}$ where $L$ is a cutoff on $u$. Then $A_{2} \sim\left(\ln \frac{L}{\varepsilon}\right)^{2}$ (see also 9]).
} 
Below we shall repeat this computation starting with a T-dual superstring action which appears in the context of the proposed relation between gluon scattering amplitudes and Wilson loops in [26]. It was suggested in [26] that using T-duality one can relate the expression for the 4-gluon scattering amplitude to the expectation value of a certain 4-cusp Wilson loop. It was argued that the world surface relevant for the 4-gluon high energy scattering can be found via 2-d duality by starting with the null cusp solution of [8], "lifting" it to global $A d S_{5}$ coordinates, applying a certain $S O(2,4)$ conformal transformation (a discrete one plus a "conformal boost" depending on $s / t$ ), and then reinterpreting the result back in the Poincare coordinates.

If we first formally ignore the issue of IR regularization, 17 we can argue that since the superstring partition function is supposed to be invariant under the global symmetry of $A d S_{5} \times S^{5}$ and, moreover, since the quantum fluctuations should not distinguish between the global and the Poincare coordinates (a change of coordinates in $A d S_{5} \times S^{5}$ is a local field redefinition of the quantum $2 \mathrm{~d}$ fields) it should not matter which form of the 1-cusp Wilson loop solution we use for the 1-loop computation.

Then assuming an "a posteriori" cutoff on the area of world surface we shall find in section 3.1 the same expression for the coefficient of the IR singular part of the partition function, confirming at strong coupling that $f(\lambda)$ that appears in (1.2) is the same universal cusp anomaly as found in the spinning closed string and the 1-cusp Wilson loop cases.

The second part of the proposal of [26] states that the counterpart of the IR dimensional regularization on the gauge theory side should be to consider the dual string theory defined in a "dimensionally regularized" version of the $A d S_{5} \times S^{5}$ background with the analog of the boundary having $D=4-2 \epsilon$ dimensions. This proposal worked fine at the level of the classical string theory, i.e. the leading order in strong coupling expansion, reproducing [26] the same structure of the 4-gluon amplitude as conjectured [24] from the resummation of the weak coupling perturbation theory.

In section 3.2 we shall explore how this "dual" version of dimensional regularization works at the quantum string level. This "built-in" IR cutoff breaks $S O(2,4)$ invariance and thus distinguishes between the 1-cusp and 4-cusp solutions, with the latter being relevant for the physical scattering amplitude case. Since it appears to be hard to find the explicit "dimensionally regularized" version of the 4-cusp solution 18 we shall limit ourselves to the case of the $D=4-2 \epsilon$ regularized version of the 1-cusp solution (which happens to be a simple generalization [26] of the solution of [8]).

Our motivation will be based on the expectation that the 1-cusp contribution should be closely related to the leading singularity of the IR divergent part (the Sudakov form factor) of the scattering amplitude, but we should admit that there is no obvious argument supporting

\footnotetext{
${ }^{17}$ By IR regularization we mean the one that regularizes the massless gluon amplitudes. In the T-dual Wilson loop picture it appears as a "UV" regularization at small values of the coordinate $z$. In addition, the area of the 1-cusp solution has also an "IR" divergence at large values of coordinates $y_{i}$. This "IR" divergence is automatically absent in the 4-cusp case, being effectively cut off by the lengths of the sides of the contour related to momentum invariants $s$ and $t[26$.

${ }^{18}$ One could attempt to use perturbation theory in $\epsilon$ (see Appendix A), and even the leading-order form of the solution was apparently enough at the classical level [26. However, it is not clear why the same should apply at the 1-loop level. There is also a technical problem that expanding the exact-in- $\epsilon$ action near a perturbative in $\epsilon$ solution will lead to spurious (off-shell) $2 \mathrm{~d}$ divergences and $\kappa$-symmetry gauge dependence.
} 
that beyond the classical string level 19

We shall then redo the same 1-loop computation as in sections 2.2 and 3.1 for the null 1-cusp solution now in "dimensionally regularized" T-dual geometry. We shall find that, contrary to what happened at the string tree level, the "dimensional regularization" prescription no longer appears to provide a natural cutoff of the result at small $z$. Moreover, even if we formally assume that the 1-loop area factor should be again regularized in exactly the same way as the tree-level one, we will still not be able to relate the 1-loop coefficient of the $\frac{1}{\epsilon^{2}}$ pole to the 1-loop coefficient in the scaling function (1.1). More generally, there appears to be a problem with the usual RG-type relation between the coefficient of the $\frac{1}{\epsilon^{2}}$ pole and the cusp anomaly when applied order by order in the inverse string tension expansion.

We shall comment on these problems in section 3.3. As we shall discuss, it is not a priori clear why considering string theory in the "dimensionally regularized" background should provide the required analog of the IR regularization of gauge theory, with the logarithm of the Wilson loop expectation value scaling as $\frac{q_{1}}{\epsilon^{2}}+\frac{q_{2}}{\epsilon}+q_{3}$ at each order in the $\alpha^{\prime} \sim \frac{1}{\sqrt{\lambda}}$ expansion 20 We shall conclude that the "dimensional regularization" prescription as formulated in [26] does not appear to work (at least in the most naive way) order by order in the strong coupling expansion. One option is that its application may require a resummation of $\frac{1}{\sqrt{\lambda}}$ expansion; alternatively, it may require some modification, for example, a modified relation between the parameters $\epsilon$ and $\mu$ on the gauge and the string sides. This important issue needs further clarification.

\subsection{1-loop correction in T-dual picture: fermionic action}

Starting with the $A d S_{5} \times S^{5}$ superstring action [31] written in a particular $\kappa$-symmetry gauge and applying $2 \mathrm{~d}$ duality along 4 isometric directions of $A d S_{5}$ in Poincare coordinates it was found in [15] that the resulting action takes a remarkably simple form $(m=0,1,2,3 ; s=$ $\left.1, \ldots, 6, \quad z^{2}=z^{s} z^{s}\right)$

$$
\tilde{S}=-\frac{\sqrt{\lambda}}{2 \pi} \int d^{2} \sigma\left[\frac{1}{2 z^{2}}\left(\partial^{a} y^{m} \partial_{a} y^{m}+\partial^{a} z^{s} \partial_{a} z^{s}\right)+2 i \epsilon^{a b} \bar{\vartheta}\left(\partial_{a} y^{m} \Gamma_{m}+\partial_{a} z^{s} \Gamma_{s}\right) \partial_{b} \vartheta\right] .
$$

Here $\vartheta$ is a Majorana-Weyl $10 \mathrm{~d}$ spinor related to the two original fermionic coordinates by a certain $y$-dependent rotation and a $\kappa$-symmetry gauge choice involving $\left(\delta^{I J} \pm \Gamma_{0123} \epsilon^{I J}\right)$. Note

\footnotetext{
${ }^{19}$ At the tree level it was argued in [26] that the single-cusp contributions determine the $\frac{1}{\epsilon^{2}}$ part of the area of the 4-cusp surface. Moreover, it was noticed in [38] that with a special choice of the "IR" cutoff on the length of the two sides of the cusp (equal to $2 \pi \sqrt{-s}$ where $s$ is the Mandelstam variable) which complements the "UV" dimensional regularization at small $z$ one gets exactly the same answer for the two leading singular terms $\frac{1}{\epsilon^{2}}$ and $\frac{1}{\epsilon}$ in the 1-cusp area as found in the area for the full 4-cusp surface in [26. It is not clear, however, why this observation should extend to the quantum string case.

${ }^{20}$ At the same time, one can give a general argument that the logarithm of the relevant IR singular factor in the perturbative gauge theory amplitude in $D=4-2 \epsilon$ should have this particular structure order by order in $\lambda[22,24$.

${ }^{21}$ Here we assume conformal gauge and thus ignore the dilaton coupling originating from the $2 \mathrm{~d}$ duality transformation. We also use Minkowski signature on the world sheet.
} 
that the action (3.1) is exactly quadratic in fermions 22 This action was interpreted in [15] as describing a fundamental superstring propagating in the background which is T-dual to the near-core D3-brane one, i.e. in the near-core smeared D-instanton [36] background.

Below we shall first rederive the fermionic part of (3.1) by starting with the general form of the quadratic part of the GS action in a type IIB superstring background and specifying it to the case of the smeared D-instanton background. We shall then expand that action near the homogeneous cusp solution (2.19),(2.28) and show that the resulting fermionic spectrum is the same as found above in section 2.3 from the quadratic part of the original $A d S_{5} \times S^{5}$ action (2.37). This is of course expected on the basis that the two actions are related by the $2 \mathrm{~d}$ duality transformation. The resulting 1-loop partition function is then the same as in (2.40) and thus it leads to the same 1-loop term in the coefficient function $f(\lambda) 23$

To quadratic order in fermions the form of the GS superstring action is determined by the generalized covariant derivative that enters the variation of the target space gravitino field. The fact that we consider a superstring propagating in a background which is a type II supergravity solution guarantees the $\kappa$-symmetry of the classical string action [37]. We shall use the normalization in which the bosonic part of the string-frame type IIB supergravity Lagrangian is

$$
\mathcal{L}_{\mathrm{IIB}}=e^{-2 \phi}\left[R+4(\partial \phi)^{2}-\frac{1}{12} H_{M N K}^{2}\right]-\frac{1}{2} F_{M}^{2}-\frac{1}{12} F_{M N K}^{2}-\frac{1}{4 \times 5 !} F_{M N K L P}^{2}
$$

and the gravitino supersymmetry transformation rule is $(I, J=1,2)$

$$
\begin{aligned}
\delta \psi_{M}^{I} & =\mathrm{D}_{M}^{I J} \varepsilon^{J} \\
\mathrm{D}_{M} & =\left(\partial_{M}+\frac{1}{4} \omega_{M}{ }^{A B} \Gamma_{A B}\right)-\frac{1}{8} s_{3} H_{A B M} \Gamma^{A B}+\frac{1}{8} e^{\phi}\left[\mathrm{F}_{(1)} s_{0}+\mathrm{F}_{(3)} s_{1}+\frac{1}{2} \mathrm{~F}_{(5)} s_{0}\right] \Gamma_{M}
\end{aligned}
$$

with the $2 \times 2$ matrices $s_{3}=\sigma_{3}, s_{1}=\sigma_{1}, s_{0}=i \sigma_{2}$ and $\mathrm{F}_{(n)}=\frac{1}{n !} F_{A_{1} \ldots A_{n}} \Gamma^{A_{1} \ldots A_{n}}$. Then the quadratic fermionic action is a generalization of (2.37)

$$
L_{F 2}=i\left(\eta^{a b} \delta^{I J}-\epsilon^{a b} \mathrm{~s}^{I J}\right) \bar{\theta}^{I} \phi_{a} \mathrm{D}_{b}^{I J} \theta^{J}
$$

For $1 / 2$ supersymmetric supergravity backgrounds one has $\mathrm{D}^{2}=0$ (i.e. $\left[\mathrm{D}_{M}, \mathrm{D}_{N}\right]=0$ ) which is the condition of integrability of the Killing spinor equation; shifting $\theta$ by a Killing spinor is then a global fermionic symmetry of the GS action.

While in this subsection we are ultimately interested in the case when we start with a D3brane solution with $D=4$ longitudinal directions, in the next section we will consider, following [26], the case when $D=4-2 \epsilon, \epsilon \rightarrow 0$, so let us keep $D$ arbitrary for generality. The solution Tdual to a Dp-brane solution (assuming T-duality is formally applied in all $D=p+1$ longitudinal directions) is the D-instanton [36] smeared in $D$ directions

$$
d s_{10}^{2}=H^{1 / 2}(z)\left(d y_{D}^{2}+d z_{10-D}^{2}\right)
$$

\footnotetext{
${ }^{22}$ The starting point in 15 was the action in a special $\kappa$-symmetry gauge choice found in 34 . An equivalent action also becoming quadratic in fermions (and having the same structure as (3.1)) after the T-duality along the 4 Poincare patch coodinates was found in another $\kappa$-symmetry "S-gauge" in Appendix C of [35].

${ }^{23}$ As a by-product, we will thus provide a direct check of the 1-loop finiteness of the action (3.1).
} 


$$
\begin{aligned}
e^{\phi} & =H, \quad F_{(1)}=d C, \quad C=i H^{-1}, \quad e^{\phi} F_{(1)}=-i d \ln H, \\
\nabla_{10-D}^{2} H & =0 \rightarrow H=\frac{c_{D} R^{4} \tilde{\mu}^{4-D}}{z^{8-D}}, \quad R^{4}=\lambda \alpha^{\prime 2} .
\end{aligned}
$$

Here the harmonic function $H$ was taken in the near-core limit and $\tilde{\mu}$ is an analog of the gauge theory renormalization scale in dimensional regularization 24 Note that the original $\mathrm{RR}$ scalar $C$ is imaginary for the D-instanton solution 25

The corresponding $\kappa$-invariant quadratic fermion Lagrangian (3.4) then takes the form (cf. (2.37)):

$$
L_{F 2}=i\left(\eta^{a b} \delta^{I J}-\epsilon^{a b}{ }_{\mathrm{S}}^{I J}\right) \bar{\theta}^{I} \phi_{a}\left[\left(\partial_{b}+\frac{1}{4} \omega_{b}{ }^{A B} \Gamma_{A B}\right) \delta^{J K}-\frac{1}{8} i \epsilon^{J K} e^{\phi} \mathrm{F}_{(1)} \phi_{b}\right] \theta^{K} .
$$

Using that the only nonzero components of the connection for the metric in (3.5) are

$$
\omega_{M}^{A i}=-\omega_{M}^{i A}=\delta_{M}^{A} \partial_{i} \ln H^{1 / 4}, \quad i=9-D, \ldots, 9,
$$

it is easy to show that

$$
\begin{array}{r}
L_{F 2}=i\left(\eta^{a b} \delta^{I J}-\epsilon^{a b}{ }^{I J}\right) H^{1 / 4} \partial_{a} x^{A} \bar{\theta}^{I} \Gamma_{A}\left[\left(\partial_{b}+\frac{1}{2} \partial_{b} \ln H^{1 / 4}\right) \delta^{J K}\right. \\
\left.+\frac{1}{2} \partial_{b} x^{B} \partial_{i} \ln H^{1 / 4} \Gamma^{i} \Gamma_{B}\left(\delta^{J K}-i \epsilon^{J K}\right)\right] \theta^{K}
\end{array}
$$

where $i=9-D, \ldots, 9, x^{A}=\left(y^{m}, z^{s}\right)$, and $A, B=0, \ldots, 9$ are flat indicies.

We can eliminate the first abelian connection term $\left(\partial_{a}+\frac{1}{2} \partial_{a} \ln H^{1 / 4}\right)$ by redefining

$$
\theta^{I}=H^{-1 / 8} \Theta^{I}
$$

thus getting

$$
\begin{aligned}
L_{F 2}=i\left(\eta^{a b} \delta^{I J}-\right. & \left.\epsilon^{a b}{ }^{I J}\right) H^{1 / 4} \partial_{a} x^{A} \bar{\theta}^{I} \Gamma_{A}\left[\partial_{b} \delta^{J K}\right. \\
+ & \left.\frac{1}{2} \partial_{b} x^{B} \partial_{i} \ln H^{1 / 4} \Gamma^{i} \Gamma_{B}\left(\delta^{J K}-i \epsilon^{J K}\right)\right] \theta^{K} .
\end{aligned}
$$

Next, we note that the only difference between this action and the one for the $A d S_{5} \times S^{5}$ supported by the 5 -form flux is that the composite connection term $\partial_{b} x^{B} \partial_{i} \ln H^{1 / 4} \Gamma_{i} \Gamma_{B}\left(\delta^{J K}-\right.$ $\left.i \epsilon^{J K}\right)$ is larger by a factor of $\frac{8-D}{4}$ (i.e. by $1+\frac{1}{2} \epsilon$ if $D=4-2 \epsilon$ ). In the absence of regularization, i.e. for $\epsilon=0$ this connection term can be eliminated as in [15] by a rotation of fermions by the same matrix that appears in the solution of the Killing spinor equation. The same transformation can be made also for generic $D 26$

$$
\Theta^{I}=\left(\Lambda^{\frac{8-D}{4}}\right)^{I J} \vartheta^{J}
$$

\footnotetext{
${ }^{24}$ In the notation of [26], $c_{D}=2^{4 \epsilon} \pi^{3 \epsilon} \Gamma(2+\epsilon), \tilde{\mu}=\frac{\mu}{\sqrt{4 \pi e^{-\gamma}}}$ and $\alpha^{\prime}=1$.

${ }^{25}$ The dual to $F_{(1)}$ form $F_{(9)}$ is real in euclidean signature case. Let us note also that one may check that the dilatino variation $\left(\delta \lambda=\frac{1}{2} \not \partial \phi \varepsilon+\frac{1}{2} e^{\phi} \mathrm{F}_{(1)} s_{0} \varepsilon+\right.$ other fields) vanishes if $\varepsilon^{1}=-\varepsilon^{2}$ and that the same relation annihilates the gravitino variation.

${ }^{26}$ The spinor matrix $\Lambda$ here is a function of $S^{5}$ coordinates which appears in the expression for the Killing spinors on $S^{5}$.
} 
Consequently, the action becomes simple when written in terms of $\vartheta^{I}$.

We are still to fix the $\kappa$-symmetry gauge. Ref. [15] used in the original D3-brane context the gauge $\left(\delta^{I J}-\Gamma_{0123} \epsilon^{I J}\right) \vartheta^{I}=0$ which amounts to setting $\vartheta^{1}=\Gamma_{0123} \vartheta^{2}$. The equivalent action is found by choosing, as appropriate in the D-instanton context $\left(\Gamma_{11}^{2}=1\right)$

$$
\vartheta^{1}=\Gamma_{11} \vartheta^{2}, \quad \text { i.e. } \quad \vartheta^{1}=\vartheta^{2} \equiv \vartheta \text {, }
$$

where we used that the type IIB fermions are Majorana-Weyl with the same chirality 27 This finally leads to the same fermionic Lagrangian as in (3.1)

$$
L_{F 2}=-2 i \epsilon^{a b} \partial_{a} x^{A} \bar{\vartheta} \Gamma_{A} \partial_{b} \vartheta .
$$

The $2 \mathrm{~d}$ duality derivation of (3.1) in [15] implies that the superstring action does not actually contain any higher-order fermionic terms.

Finally, we are ready to return to the problem of computing the 1-loop correction in "T-dual" string theory expanded near the null cusp solution (2.15) (or (2.19) or (2.28)). The bosonic part of the action is the same $A d S_{5} \times S^{5}$, so the fluctuation spectrum is the same as in section 2.2. To find the corresponding fermionic fluctuation spectrum it is easiest to go back to the "unrotated" form of the Lagrangian (3.10) and fix the $\theta^{1}=\theta^{2}$ gauge. We should also take into account that we are now interested in the euclidean world-sheet metric, i.e. we are to replace $\eta_{a b} \rightarrow \delta_{a b}, \epsilon_{a b} \rightarrow i \epsilon_{a b}$. The resulting fermionic action has then constant coefficient:28 and one finds 8 fermionic massive modes with mass 1 , i.e. exactly the same spectrum as in (2.40). Using a "direct" IR regularization of the world-sheet area as in 8] or in section 2.2 (with $\ln ^{2} \mu \sim \gamma \ln \frac{L}{\ell}$ ) we then reproduce the first universal term in the amplitude (1.2) with the same function $f(\lambda)$ as in the cusp anomaly.

\subsection{1-loop correction in T-dual picture with dimensional regulariza- tion}

Let us now try to generalize the discussion at the classical string level in [26] and consider the dimensionally regularized version of the 1-loop computation of the T-dual 1-cusp Wilson loop expectation value described in the previous subsection.

In the original high-energy scattering set-up we should replace the $A d S_{5} \times S^{5}$ space by the near-core D-brane solution with the total number of longitudinal directions being $D=4-2 \epsilon$, i.e. having the metric

$$
d s_{10}^{2}=H^{-1 / 2}(z) d x_{D}^{2}+H^{1 / 2}(z)\left(d z^{2}+z^{2} d \Omega_{9-D}^{2}\right)
$$

\footnotetext{
${ }^{27}$ There is a subtlety in the discussion of the GS action in D-instanton background in that we should have considered the euclidean target space and thus formally complexify the fermions (cf. [36]). In particular, the rotation needed to eliminate the second line in (3.10) is complex and the natural gauge choice appears to be $\theta_{1}=i \theta_{2}$.

${ }^{28}$ Starting with the form of the action in (3.13) to get the simple fermionic fluctuation Lagrangian one would need to "undo" the rotation (3.11), i.e. to apply a transformation $U=\cosh \eta+\sinh \eta \Gamma_{0} \Gamma_{1}$ with $\eta$ being linear in world-sheet coordinates.
} 
which should be supported by the corresponding dilaton and RR field strength. To consider the number of longitudinal dimensions $D$ to be continuous may appear a bit odd since the $1 / 2$ supersymmetric type IIB Dp-brane solutions exist only for even integer $D$. This problem goes away after we T-dualize along the $D$ directions $x^{m} \rightarrow y^{m}$ in order to switch to the "momentum-space" Wilson-loop description [26]. This leads to the smeared D-instanton background already given in (3.5). Ignoring the overall string tension factor $T_{\epsilon}=\frac{\sqrt{c_{D} \lambda \tilde{\mu}^{2 \epsilon}}}{2 \pi}$ coming from the numerator of $H$ in $(3.5)$ we then get the following dual metric

$$
\tilde{d s}_{10}^{2}=z^{-\epsilon}\left[\frac{d y_{4-2 \epsilon}^{2}+d z^{2}}{z^{2}}+d \Omega_{5+2 \epsilon}^{2}\right]
$$

In the strict $\epsilon \rightarrow 0$ limit we get back to the $A d S_{5} \times S^{5}$ metric where the $A d S$ part (cf. (2.4 ) and the internal sphere part factorize. This factorization will still be true at the classical level (assuming, as we will, that one is interested in solutions localized in $S^{5+2 \epsilon}$ ), but it will no longer hold at the level of the quantum fluctuation.

Let us start with generalizing the light-like cusp solution (2.15) from the case of the $A d S_{5}$ metric (2.13) to the case of the "regularized" metric (3.15). Assuming that the only non-zero coordinates are again $z, u, \xi$ (in the notation of (2.13) ), choosing the static gauge with $(u, \xi)$ as the world-sheet directions and making the $\xi$-homogeneous ansatz for $z$, i.e. $z=F(u)$, we find that the string action is proportional to $\int d \xi d u u F^{-2-\epsilon} \sqrt{F^{\prime 2}-1}$. This leads to the solution [26]

$$
z=F(u)=\sqrt{2+\epsilon} u,
$$

which generalizes the solution (2.14) of [8]. The induced metric is then

$$
d s_{2}^{2}=\frac{1+\epsilon}{(2+\epsilon)^{1+\frac{1}{2} \epsilon}}\left[\frac{d u^{2}}{u^{2+\epsilon}}+\frac{1}{1+\epsilon} \frac{d \xi^{2}}{u^{\epsilon}}\right] .
$$

Changing the coordinates, it can then be put into the standard conformal-gauge form, i.e. conformal to $d \tau^{2}+d \sigma^{2}=d \zeta d \bar{\zeta}$. In general, one can show that given any function

$$
h=h(\zeta), \quad \zeta=\tau+i \sigma,
$$

the background

$$
z=\sqrt{2+\epsilon} u, \quad u=e^{-\frac{1}{2}(h+\bar{h})}, \quad \xi=-\sqrt{1+\epsilon} \frac{h-\bar{h}}{2 i}
$$

is a conformal-gauge solution, i.e. it solves the string equations and the euclidean version of the conformal constraints. The simplest choice which is a direct generalization of the $\epsilon=0$ solution (2.15) is found if $h$ is linear in $\zeta$, i.e. $h=-\sqrt{2} \zeta$ :

$$
z=\sqrt{2+\epsilon} u, \quad u=e^{\sqrt{2} \tau}, \quad \xi=\sqrt{2(1+\epsilon)} \sigma .
$$

\footnotetext{
${ }^{29}$ Note that dimensions are balanced in the string action provided $\lambda$ is dimensionless and $y$ and $z$ have the same dimension as $\mu$, i.e. the mass dimension (this was the implicit assumption in [26]). This dimension assignment can be reversed by rescaling by a power of $\alpha^{\prime}$.
} 
Then the induced metric is conformally-flat (becoming flat for $\epsilon=0$ ) 30

$$
d s_{2}^{2}=N_{\epsilon} e^{-\sqrt{2} \epsilon \tau}\left(d \tau^{2}+d \sigma^{2}\right), \quad \quad N_{\epsilon}=\frac{2^{-\frac{1}{2} \epsilon}(1+\epsilon)}{\left(1+\frac{1}{2} \epsilon\right)^{1+\frac{1}{2} \epsilon}} .
$$

The value of the classical (euclidean) action is regularized by $\epsilon<0$ at $\tau \rightarrow-\infty$ but is still divergent in other limits, i.e. it depends on a choice of a region in $\tau, \sigma$ space. Alternatively, the action can be computed in the static gauge as in eq.(3.29) in [26], i.e. we start with $I=T_{\epsilon} N_{\epsilon} \int d \tau d \sigma e^{-\sqrt{2} \epsilon \tau}$ and rewrite it in terms of

$$
y_{ \pm}=u e^{ \pm \xi}=e^{\sqrt{2}[\tau \pm \sqrt{1+\epsilon} \sigma]}
$$

ending up with

$$
I=T_{\epsilon} K_{\epsilon} \int_{0}^{u_{*}} \frac{d y_{+} d y_{-}}{2\left(2 y_{+} y_{-}\right)^{1+\frac{1}{2} \epsilon}}, \quad T_{\epsilon}=\frac{\sqrt{c_{D} \lambda \tilde{\mu}^{2 \epsilon}}}{2 \pi}, \quad K_{\epsilon}=\frac{\sqrt{1+\epsilon}}{\left(1+\frac{1}{2} \epsilon\right)^{1+\frac{1}{2} \epsilon}},
$$

where one needs to assume a cutoff $y_{ \pm} \leq u_{*}$ at large $y_{ \pm}$which amounts to specifying the lengths of the null lines forming the boundary of the cusp surface 31 As was noticed in [38], choosing this cutoff as $u_{*}=2 \pi \sqrt{-s}$ one gets (after formally combining the 4 1-cusp contributions) the same answer for the two leading singular terms as found in the area for the full 4-cusp surface in $[26]^{32}$. Then we get from (3.23)

$$
I=\frac{1}{\epsilon^{2}} \frac{\sqrt{\lambda}}{2 \pi} \sqrt{\frac{\mu^{2 \epsilon}}{|s| \epsilon}}+\frac{1}{\epsilon} \frac{\sqrt{\lambda}}{4 \pi}(1-\ln 2) \sqrt{\frac{\mu^{2 \epsilon}}{|s|^{\epsilon}}}+\mathcal{O}\left(\epsilon^{0}\right) .
$$

Our aim now will be to compute the 1-loop correction to the $\epsilon$-regularized 1-cusp Wilson loop in the conformal gauge by expanding near (3.20). To study the bosonic fluctuations it is useful first to change the coordinates in (3.15) from $(z, u, y)$ to $\left(\nu, \beta, y^{\prime}\right)$ as follows:

$$
z=\sqrt{2+\epsilon} e^{\nu}, \quad u=e^{\nu+\beta}, \quad y_{k}=e^{\nu} y_{k}^{\prime} .
$$

Then the 10-d metric (3.15) takes the form

$$
d s_{10}^{2}=e^{-\epsilon \nu}\left((2+\epsilon)^{-1-\frac{1}{2} \epsilon}\left[-e^{2 \beta}(d \nu+d \beta)^{2}+e^{2 \beta} d \xi^{2}+(2+\epsilon) d \nu^{2}+\left(d y^{\prime}+d \nu y^{\prime}\right)_{2-2 \epsilon}^{2}\right]\right.
$$

\footnotetext{
${ }^{30}$ The choice of $h$ that corresponds to flat induced metric is $h(\zeta)=\frac{2}{\epsilon} \ln \left[1+\frac{\epsilon}{2} \frac{(2+\epsilon)^{(2+\epsilon) / 4}}{(1+\epsilon)^{1 / 2}} \zeta\right]$.

${ }^{31}$ Our notation for $\tau, \sigma$ differs from eq. [26] by $\sqrt{2}$ factor; $I$ is euclidean action, i.e. $I=-i S$ in the notation of [26]. The $1 / 2$ factor in the integral over $y_{ \pm}$comes from from the Jacobian.

${ }^{32}$ As was noted in [26, to get this singular part of area one actually needs only the $\epsilon=0$ form of the classical solution. Ignoring the $\epsilon$-dependence in (3.20), the restriction of $\left(y_{+}, y_{-}\right)$to the square $\left[\left(0, u_{*}\right),\left(0, u_{*}\right)\right]$ can be easily translated into the domain in $(\tau, \sigma)$ space using that $y_{ \pm}=e^{\sqrt{2}(\tau \pm \sigma)}$. Integrating the square root of the determinant of the induced metric (3.21) in this region will lead to the same expression for the area as in the $y_{ \pm}$coordinates in (3.23).
} 


$$
\left.+(2+\epsilon)^{-\frac{1}{2} \epsilon} d \Omega_{5+2 \epsilon}^{2}\right),
$$

where $y_{k}^{\prime}$ stand for the rest of the "longitudinal" coordinates of the metric. Since the 1-cusp solution is localized in these coordinates and also in the sphere coordinates, the corresponding parts of the quadratic fluctuation Lagrangian will contain two sets of $2-2 \epsilon\left(y_{k}^{\prime}\right)$ and $5+2 \epsilon$ $\left(Y_{s}\right)$ decoupled massive modes.

Note that since for $\epsilon=0$ the coordinates $\nu$ and $\xi$ are isometric angles in this parametrization, this explains why the 1-cusp solution was homogeneous. Indeed, the solution (3.20), i.e.

$$
\nu=\sqrt{2} \tau, \quad \xi=\sqrt{2(1+\epsilon)} \sigma, \quad \beta=0
$$

has $\nu$ and $\xi$ linear in $\tau$ and $\sigma$.

This homogeneity is apparently broken for $\epsilon \neq 0$ by the overall $e^{-\epsilon \nu} \sim z^{-\epsilon}$ factor in the metric (3.26). Remarkably, it can be effectively regained at the level of the quadratic fluctuations if we properly redefine the fluctuation fields when expanding near the classical solution (3.27)

$$
\begin{aligned}
& \nu=\sqrt{2} \tau+e^{\frac{\epsilon}{\sqrt{2}} \tau} \tilde{\nu}, \quad \beta=e^{\frac{\epsilon}{\sqrt{2}} \tau} \tilde{\beta}, \quad \xi=\sqrt{2+2 \epsilon}\left(\sigma+e^{\frac{\epsilon}{\sqrt{2}} \tau} \tilde{\xi}\right), \\
& y_{k}^{\prime}=e^{\frac{\epsilon}{\sqrt{2}} \tau} \tilde{y}_{k}, \quad Y_{s}=e^{\frac{\epsilon}{\sqrt{2}} \tau} \tilde{Y}_{s}, \quad k=1, \ldots, 2-2 \epsilon, \quad s=1, \ldots, 5+2 \epsilon .
\end{aligned}
$$

The euclidean-signature quadratic fluctuation Lagrangian in the conformal gauge will then have constant coefficients. Indeed, it is given by (up to integration by parts and trivial rescalings)

$$
\begin{aligned}
\tilde{L}_{2}= & \frac{1}{2}(1+\epsilon) \partial^{a} \tilde{\xi} \partial_{a} \tilde{\xi}+\frac{1}{2}(1+\epsilon) \partial^{a} \tilde{\nu} \partial_{a} \tilde{\nu}-\frac{1}{2} \partial^{a} \tilde{\beta} \partial_{a} \tilde{\beta}-\partial^{a} \tilde{\beta} \partial_{a} \tilde{\nu} \\
& +2 \sqrt{2}\left[\left(1+\frac{1}{2} \epsilon\right) \tilde{\beta} \partial_{\tau} \tilde{\nu}-(1+\epsilon) \tilde{\beta} \partial_{\sigma} \tilde{\xi}+\frac{\epsilon}{2}(1+\epsilon) \tilde{\nu} \partial_{\sigma} \tilde{\xi}\right] \\
& +\frac{\epsilon^{2}}{4}(1+\epsilon) \tilde{\xi}^{2}+\frac{\epsilon^{2}}{4}(1+\epsilon) \tilde{\nu}^{2}-\frac{\epsilon^{2}}{4} \tilde{\beta}^{2}-\frac{1}{2} \epsilon(4+3 \epsilon) \tilde{\nu} \tilde{\beta} \\
& +\frac{1}{2} \partial^{a} \tilde{y}_{k} \partial_{a} \tilde{y}_{k}+\left(1+\frac{1}{2} \epsilon\right)^{2} \tilde{y}_{k}^{2}+\frac{1}{2} \partial^{a} \tilde{Y}_{s} \partial_{a} \tilde{Y}_{s}+\frac{\epsilon^{2}}{4} \tilde{Y}_{s}^{2} .
\end{aligned}
$$

For $\epsilon=0$ this Lagrangian becomes equivalent to (2.36) (with $S^{5}$ modes added).

Thus even for $\epsilon \neq 0$, the 1-cusp solution is again effectively homogeneous, and, as in the discussion of the non-regularized case in (2.42), we should then find that the 1-loop quantum correction is again proportional to (regularized value of) the world-sheet area.

It is, however, clear from the structure of the metric (3.26) that once we go beyond the quadratic fluctuation (string 1-loop) level the interaction vertices will contain powers of the effective $\tau$-dependent coupling proportional to the inverse of the "running string tension", i.e. $\frac{1}{\sqrt{\lambda}} z^{\epsilon} \sim \frac{1}{\sqrt{\lambda}} e^{\epsilon \nu}=\frac{1}{\sqrt{\lambda}} e^{\sqrt{2} \epsilon \tau}$. While this effective string tension provided a cutoff at small $z$ (large negative $\tau$ ) at the string tree level, i.e. in the world-sheet area, this apparently will no longer be so at higher orders of inverse tension expansion. The same conclusion is then expected also in the case of the 4-cusp solution of [26]. Thus there appears to be a problem with implementation of the idea of this "dimensional regularization", at least order by order in $\frac{1}{\sqrt{\lambda}}$ expansion. We shall return to the discussion of this issue below.

Given that the action (3.29) has constant coefficients it is straightforward to find the fluctuation spectrum. We shall first assume that $\tau$ and $\sigma$ run in the infinite range, i.e. ignore the 
presence of a cutoff on the world-sheet coordinates that implements an "IR" cutoff in spacetime (we will need to introduce it at the end to regularize the area factor as in the 1-cusp case in (2.42) $)$. We shall also assume trivial boundary conditions on the $2 \mathrm{~d}$ fluctuation fields. Then we can use the standard $2 \mathrm{~d}$ momentum representation to diagonalize the fluctuation modes 33

The three mixed modes $\tilde{\xi}, \tilde{\nu}, \tilde{\beta}$ in (3.29) lead to the following contribution to the 1-loop effective action, i.e. to the $1 / 2$ of the logarithm of the fluctuation determinant (here $p^{2}=p_{1}^{2}+p_{2}^{2}$; cf. $(2.39),(2.40))$

$$
\frac{1}{2} V_{2} \int \frac{d^{2} p}{(2 \pi)^{2}}\left(\ln \left[p_{1}^{2}+\left(p_{2}+\frac{1}{2} \epsilon\right)^{2}\right]+\ln \left[p_{1}^{2}+\left(p_{2}-\frac{1}{2} \epsilon\right)^{2}\right]+\ln \left[p^{2}+4+4 \epsilon+\frac{1}{2} \epsilon^{2}\right]\right) .
$$

The contribution of the first two terms here is equivalent to the contribution of the two massless modes, i.e. is the same as in the $\epsilon=0$ case. Indeed, using polar coordinates in $p$-space and integrating over the angle using $\int_{0}^{2 \pi} d \theta \ln (a+b \cos \theta)=2 \pi \ln \left(\frac{a}{2}+\frac{1}{2} \sqrt{a^{2}-b^{2}}\right)$ we find that

$$
\int d^{2} p\left(\ln \left[p_{1}^{2}+\left(p_{2}+\frac{1}{2} \epsilon\right)^{2}\right]+\ln \left[p_{1}^{2}+\left(p_{2}-\frac{1}{2} \epsilon\right)^{2}\right]\right)=2 \int d^{2} p \ln p^{2} .
$$

This is exactly what is required to cancel the contribution of the two massless modes of the diffeomorphism ghosts in the conformal gauge.

The action needed to find the fermionic contribution was already discussed in subsection 3.1. Starting with the action (3.10) where $H$ is now given by (3.5) with $D=4-2 \epsilon$, one should plug in there the solution (3.20) and repeat the same computation of the fermionic fluctuation frequencies as described at the end of the previous subsection. Using the $\theta^{1}=\theta^{2}$ gauge and noticing that the abelian connection term drops out since $\bar{\theta} \Gamma^{A} \theta=0$, it follows that the quadratic fermion Lagrangian is

$$
\mathcal{L}=2 \sqrt{2} i u\left[\bar{\theta}\left(\Gamma_{0}+\sqrt{2+\epsilon} \Gamma_{1}\right) \partial_{0} \theta+\sqrt{1+\epsilon} \bar{\theta} \Gamma_{2} \partial_{1} \theta-\sqrt{\frac{1}{2}(1+\epsilon)(2+\epsilon)} \bar{\theta} \Gamma_{012} \theta\right]
$$

The overall factor of $u$ can be eliminated by rescaling of $\theta$ by $u^{1 / 2}$. The fermion propagator is then proportional to the inverse of $p^{2}+\left(1+\frac{1}{2} \epsilon\right)$ implying that we find 8 fermionic modes with mass squared equal to $1+\frac{1}{2} \epsilon$.

Including the contribution of the $2-2 \epsilon$ longitudinal modes $\tilde{y}_{k}$ and $5+2 \epsilon$ sphere modes $\tilde{Y}_{s}$ in (3.29) we end up with the following expression for the 1-loop effective action generalizing the

\footnotetext{
${ }^{33} \mathrm{~A}$ clarification is in order. When discussing a spectrum of quadratic fluctuations of a string sigma model we may assume that fluctuations of the coordinates are normalized as $\int d^{2} \sigma \sqrt{\bar{g}} \hat{x}^{M} \hat{x}^{N} G_{M N}(x)$. The redefinition of the fluctuations made in (3.28) removes the $G_{M N}(x)$ factor, i.e. replaces $\hat{x}^{M}$ by the tangent-space vectors $\tilde{x}^{M}$. The fiducial $2 \mathrm{~d}$ metric $\bar{g}_{a b}$ may be either the original independent metric of Polyakov's action or the induced metric (on the equations of motion or in the conformal gauge they differ only by a conformal factor). Since the induced metric in (3.21) is not flat for $\epsilon \neq 0$ one may wonder if we are allowed to consider the standard Fourier-mode basis when computing the spectrum. The answer is yes, provided all the modes (including the fermions and the conformal gauge ghosts) are normalized using the same fiducial metric: its conformal factor dependence should then cancel out since the theory should not have nontrivial Weyl anomaly (see also [17]).
} 
one in (2.39), (2.40) to the case of $\epsilon \neq 0$

$$
\begin{aligned}
& \Gamma_{1}=\frac{1}{2} V_{2} \int \frac{d^{2} p}{(2 \pi)^{2}}\left(\ln \left[p^{2}+4+4 \epsilon+\frac{1}{2} \epsilon^{2}\right]+(2-2 \epsilon) \ln \left[p^{2}+2\left(1+\frac{1}{2} \epsilon\right)^{2}\right]\right. \\
& \left.+(5+2 \epsilon) \ln \left[p^{2}+\frac{1}{2} \epsilon^{2}\right]-8 \ln \left[p^{2}+1+\frac{1}{2} \epsilon\right]\right) .
\end{aligned}
$$

As expected, this expression is $2 \mathrm{~d}$ UV finite (the degrees of freedom and mass sum rules $\sum_{i} n_{i}=$ $0, \quad \sum_{i} n_{i} M_{i}^{2}=0$ are satisfied) 44 for any $\epsilon$ : the D-instanton background (3.5) we started with is $\kappa$-symmetric and thus the GS superstring action should be 1-loop finite.

The integral over the 2-momentum can be computed explicitly and we get (with $V_{2}=2 A_{2}$ as appropriate for a single cusp case as in (2.41))

$$
\begin{gathered}
\Gamma_{1}=\frac{1}{4 \pi} c_{1}(\epsilon) A_{2} \\
c_{1}(\epsilon)=\int_{0}^{\infty} d v\left(\ln \left[v+4+4 \epsilon+\frac{1}{2} \epsilon^{2}\right]+2(1-\epsilon) \ln \left[v+2\left(1+\frac{1}{2} \epsilon\right)^{2}\right]\right. \\
\left.+(5+2 \epsilon) \ln \left[v+\frac{1}{2} \epsilon^{2}\right]-8 \ln \left[v+1+\frac{1}{2} \epsilon\right]\right) \\
=-\frac{1}{2}\left(8+8 \epsilon+\epsilon^{2}\right) \ln \left(8+8 \epsilon+\epsilon^{2}\right)+2 \epsilon\left(2+3 \epsilon+\epsilon^{2}\right) \ln (2+\epsilon)-\epsilon^{2}(5+2 \epsilon) \ln |\epsilon| .
\end{gathered}
$$

Expanding for $|\epsilon| \rightarrow 0$ gives

$$
c_{1}(\epsilon)=-12 \ln 2-4(1+2 \ln 2) \epsilon+\frac{1}{2}(-1+9 \ln 2) \epsilon^{2}-5 \epsilon^{2} \ln |\epsilon|+\mathcal{O}\left(\epsilon^{3}\right) .
$$

For $\epsilon=0$ we reproduce the value of $a_{1}=\frac{1}{4 \pi} c_{1}(0)$ in (2.41). Note that the $\epsilon^{2} \ln |\epsilon|$ term here originates from the $S^{5}$ modes that were massless in the $D=4$ case.

The 2-d area $A_{2}$ that factorizes here as in (2.39) due to the effective homogeneity of the background is simply $\frac{1}{2} \int d \tau d \sigma$, i.e. it does not contain the $\frac{1}{z^{\epsilon}} \sim e^{-\sqrt{2} \epsilon \tau}$ (the effective string tension) factor that was present in the classical expression for the area (3.23). Thus we will need to impose both "UV" and "IR" cutoffs as in the 1-cusp solution [8] in (2.42). Assuming a similar conclusion will also apply to the full 4-cusp solution this indicates that the "dimensional regularization" prescription of [26] fails to regularize the 1-loop string correction to the logarithm of the dual Wilson loop 35

\footnotetext{
${ }^{34}$ This implies also that the result cannot depend on a mass scale or the size of the "box" in which $\tau$ and $\sigma$ take values, i.e. it can depend only of ratios of masses or scales.

${ }^{35}$ It is interesting to note that the fact that in the absence of regularization the 1-cusp and 4-cusp solutions are related [26] by the transformation (2.29) combined with a "conformal boost" imply that the calculation above applies to the 4-cusp Wilson loop as well, just that the regulator factor being used in the metric is not the one of [26]. Indeed, it is not hard to find the image of $\frac{1}{z^{\epsilon}}$ through the transformations relating the 1- and 4 -cusp solutions. Thus, given the 1-cusp solution for the Lagrangian $\frac{1}{z^{2+\epsilon}}\left(d z^{2}+d y^{2}\right)$ one may construct (through the $S O(2,4)$ transformations that work at $\epsilon=0)$ a 4-cusp solution for the Lagrangian $\frac{R^{\epsilon}}{z^{2}}\left(d z^{2}+d y^{2}\right)$ where $R$ stands for $-(2 \sqrt{2} z) /\left[1+z^{2}-2\left(y_{0}+y_{1}-y_{2}\right)-y_{0}^{2}+y_{1}^{2}+y_{2}^{2}+y_{3}^{2}\right]$. This observation reiterates the fact that the right string theory counterpart of the regularization needs to be identified for the correct singular part of the amplitudes to be reproduced correctly.
} 
Despite the redefinition (3.28) that eliminated the $\frac{1}{z^{\epsilon}}$ factor from the fluctuation action we may attempt to consider, at least at a heuristic level, a possibility that the area factor $A_{2}$ in (3.34) should still be computed using the $\epsilon$-dependent induced metric in (3.21), i.e. should be taken as in (3.23), (3.24) but without 2 times the string tension factor $T_{\epsilon}$ (with extra 2 accounting for the definition of the cusp area as in (2.42), cf. also (3.24)) 36 In this case (and assuming again the momentum "IR" cutoff on the length of the cusp sides, like $u_{*}=2 \pi \sqrt{|s|}$ ) we will have from (3.23) (using tilde to indicate the result of this heuristic prescription)

$$
\tilde{A}_{2}=\frac{1}{2 \epsilon^{2}} \frac{K_{\epsilon}}{\left(2 u_{*}^{2}\right)^{\frac{1}{2} \epsilon}}=\frac{1}{2 \epsilon^{2}}-\frac{\ln \left(2 u_{*}^{2}\right)}{4 \epsilon}+\mathcal{O}\left(\epsilon^{0}\right) .
$$

Then the product of $c_{1}(3.36)$ and (3.37) in (3.34) appears to contain a surprising $\ln |\epsilon|$ term. This term should presumably be omitted: it is an artifact of the procedure of regularizing separately the area and the fluctuation determinant. In practice, one should first take $\epsilon$ to zero in $c_{1}$ and then multiply it by the divergent area, i.e. the product $\frac{1}{\epsilon^{2}} \times \epsilon^{2} \ln \epsilon^{2}$ should be set equal to zero in the $\epsilon \rightarrow 0$ limit 37 Under all these assumptions one would finish with the following expression for the singular part of the 1-loop correction

$$
\tilde{\Gamma}_{1}=-\frac{3 \ln 2}{\pi} \frac{1}{2 \epsilon^{2}}+\left[-\frac{1+2 \ln 2}{2 \pi}+\frac{3}{4 \pi} \ln 2 \ln \left(2 u_{*}^{2}\right)\right] \frac{1}{\epsilon}+\mathcal{O}\left(\epsilon^{0}\right)
$$

\subsection{Problems with IR dimensional regularization in string inverse tension expansion}

As we have pointed out above, the structure of the $D=10$ metric (3.26) with "running" effective string tension implies that the presence of the $\epsilon$ dependent factors will no longer regularize the quantum string expressions in the $z \rightarrow 0$ area beyond the classical level. A related issue is that of applicability of the $\alpha^{\prime} \sim \frac{1}{\sqrt{\lambda}}$ expansion here since the curvature of the background is singular near $z=0$ (cf. [26]).

But even assuming that some modified string theory side version of dimensional regularization prescription will lead to the $\frac{b_{1}}{\epsilon^{2}}+\frac{b_{2}}{\epsilon}+b_{3}$ contributions like (3.38) at each order in the $\frac{1}{\sqrt{\lambda}}$ expansion, we will still face the following problem in matching this strong-coupling expansion

\footnotetext{
36 An attempt to justify this suggestion could be based on the fact that the fluctuation fields in (3.29) should be normalized using the induced metric in (3.21). Then the eigenvalue problem for the fluctuation operators in (3.29) should be defined with an extra conformal factor coming from the conformally flat metric (3.21), and that may at the end produce the area factor defined with the non-flat induced metric. However, the condition of cancellation of the total Weyl anomaly means that one should be able to completely get rid of the conformal factor dependence (modulo possibly complications with boundary conditions that we are ignoring here). Consider, e.g., a 1-loop contribution of a massive scalar in curved conformally flat $2 \mathrm{~d}$ space, i.e. $\Gamma_{1}=\frac{1}{2} \ln \operatorname{det}\left(-\frac{1}{\sqrt{g}} \partial^{2}+m^{2}\right)$ or, up to a conformal anomaly term, $\frac{1}{2} \ln \operatorname{det}\left(-\partial^{2}+M^{2}\right), M^{2}=\sqrt{g} m^{2}$. Then $\Gamma_{1}$ scales as $\int d^{2} \sigma M^{2}+\ldots=\int d^{2} \sigma \sqrt{g} m^{2}+\ldots$ In our present case $M^{2}$ is actually constant, so we do not get an extra $\sqrt{g}$ factor in the integral in front of $M^{2} \ln \frac{M_{1}}{M_{2}}$ type terms in the effective action.

37 Note in particular that this issue is unrelated to the expansion of the cusp solution in small $\epsilon$ : the problem comes from the structure of the dimensionally continued metric in (3.15). This problem is more likely to be a further indication of problems with the dimensional regularization that we discuss below.
} 
with expectations based on the perturbative gauge-theory relations for the IR dimensionally regularized gluon amplitudes.

The general expression [22] for the IR singular part (1.2) of the on-shell dimensionally regularized gluon amplitude applied to the planar limit of the $\mathcal{N}=4$ SYM theory yields [24]

$$
\mathcal{A}_{\text {div }}(\mu, s)=\exp \left[-\frac{1}{8 \epsilon^{2}} f^{(-2)}(\tilde{\lambda})-\frac{1}{4 \epsilon} g^{(-1)}(\tilde{\lambda})\right], \quad \tilde{\lambda} \equiv \lambda \frac{\mu^{2 \epsilon}}{|s|^{\epsilon}},
$$

where [21, 22]

$$
f(\lambda)=\frac{d^{2}}{d \ln \lambda^{2}} f^{(-2)}(\lambda), \quad g(\lambda)=\frac{d}{d \ln \lambda} g^{(-1)}(\lambda)
$$

and $f(\lambda)$ is the cusp (soft) anomalous dimension. These relations were applied at leading strong coupling order in [26] giving the expressions for $f$ and $g$ in (1.3).

One may argue that the application of these relations at strong coupling is justified because of the finite radius of convergence of the planar weak coupling perturbation theory. The latter suggests that the equations (3.40) should be valid in a finite disk around the origin in complex $\lambda$ space from where they may be analytically continued to the strong coupling region. It is not clear, however, why $f^{(-2)}(\lambda)$ should have a regular series expansion at large $\lambda$. It is consequently unclear whether the relations (3.40) should hold order by order in the strong coupling expansion.

Indeed, there is an apparent problem with this suggestion which appears when we consider the first subleading order in $\frac{1}{\sqrt{\lambda}}$ expansion. Given the relation between gluon amplitudes and "momentum-space" Wilson loops proposed in [26] we expect that the exponent of $A_{\text {div }}(\mu, s)$ in (3.39) should have standard string perturbative expansion, i.e. that both $f^{(-2)}$ and $g^{(-1)}$ should scale as $k_{0} \sqrt{\lambda}+k_{1}+\frac{k_{2}}{\sqrt{\lambda}}+\ldots$ when expanded at large $\lambda$. On the other hand, the same pattern of strong coupling expansion is known to apply to $f(\lambda)$ [7, 10, 12] (and should presumably be true also for $g(\lambda)$ ). This is, however, inconsistent with the relations (3.40). Indeed, to reproduce the expansion (1.1), i.e. $f(\lambda)=a_{0} \sqrt{\lambda}+a_{1}+\frac{a_{2}}{\sqrt{\lambda}}+\ldots$ using (3.40) we need to assume that $f^{(-2)}(\lambda)$ should have the following behavior at large $\lambda$

$$
f^{(-2)}(\lambda \gg 1)=4 a_{0} \sqrt{\lambda}+2 a_{1}(\ln \sqrt{\lambda})^{2}+\frac{4 a_{2}}{\sqrt{\lambda}}+\ldots
$$

Here the first term has the expected classical string form [26] but the first subleading term is $(\ln \sqrt{\lambda})^{2}$ instead of a constant 38 Such logarithmic term can not appear as a perturbative string sigma correction. One may conjecture that it originates from a resummation of all higher-order strong coupling corrections 39 Another possibility is that (3.39) with (3.40) do not actually apply directly to the strong-coupling expansion as defined by the perturbative string theory.

And vice versa, even assuming that (3.38) represents the 1-loop contributions to $f^{(-2)}(\lambda)$ and $g^{(-1)}(\lambda)$, the latter are then constant ( $\lambda$-independent) and thus, according to (3.40), do not change $f(\lambda)$ (and $g(\lambda)$ ) at all, in contradiction with (1.1).

\footnotetext{
${ }^{38}$ We can of course add also a constant and as well as $\ln \sqrt{\lambda}$ term to $f^{(-2)}(\lambda)$ as this will not change the expression for $f(\lambda)$.

${ }^{39}$ We acknowledge a discussion with J. Maldacena on this issue.
} 
One abstract possibility to reconcile the strong-coupling expansions of $f^{(-2)}(\lambda)$ and of $f(\lambda)$ while preserving their relation in (3.40) could be to redefine $\lambda$ by a constant that eliminates the constant $a_{1}$ term in $f(\lambda)$. Namely, if we shift the string tension by a constant $\sqrt{\lambda}+\frac{a_{1}}{a_{0}} \equiv \sqrt{\lambda^{\prime}}$ and identify $\lambda^{\prime}$ with the gauge-theory coupling we would have no 1-loop correction in both $f(\lambda)$ and $f^{(-2)}$. Shifting the string tension by a constant seems to lead to problems with various other comparisons between the $A d S_{5} \times S^{5}$ string theory and the $\mathcal{N}=4$ supersymmetric gauge theory but in this particular setting it may seem we have little understanding of how the two couplings should actually be related.

In appendix $\mathrm{B}$ we shall present another attempt to modify the dimensional regularization prescription of [26] by relaxing the identification between the $\epsilon$ parameters on the gauge theory and the string theory sides, which may help to resolve the above problem.

Regardless of the problems discussed above, an unwelcome feature of the string-side version of the IR dimensional regularization suggested in [26] is that instead of providing a simple modification of the known $A d S_{5} \times S^{5}$ string action (by analogy with what is done to define the dimensionally regularized gauge theory 40 it instructs us to start with a dimensionallymodified supergravity background and rederive the superstring action from scratch. This is rather daunting task beyond the quadratic level in fermions which casts doubt on a practical utility of this prescription. For example, extending the above 1-loop computations to the 2-loop string theory level would be quite complicated.

\section{Note Added:}

An interesting suggestion of how to reproduce the $(\ln \sqrt{\lambda})^{2}$ term in (3.41) (and also how to treat higher-loop orders) was suggested to us by J. Maldacena (private communication). The effective string sigma model coupling that determines the higher-loop order corrections (after we rescale the fluctuation fields as in (3.28) to put the propagator term into a canonical form) is $\frac{z^{\epsilon}}{\sqrt{\lambda}}$ which grows near $z=0$ for $\epsilon<0$. At one loop we may then cut off $z$ at a minimal value $z_{\mathrm{o}}$ where $\frac{z_{\mathrm{o}}^{\epsilon}}{\sqrt{\lambda}}$ is fixed to some given value $k$, i.e. $\ln z_{\mathrm{o}}=\frac{1}{\epsilon} \ln (k \sqrt{\lambda})$. In this case the area factor of a single cusp surface which we got in (3.34) written in the same way as in (3.23) can be regularized as follows (ignoring terms of subleading orders in $\epsilon$ ): $A_{2}=\frac{1}{2} \int d \tau d \sigma \rightarrow \frac{1}{2} \int \frac{d y_{+} d y_{-}}{2\left(2 y_{+} y_{-}\right)}$, where the integral should be cut off at large $y_{ \pm}$at $u_{*}$ and small $y_{ \pm}$by the above cutoff at small z. Namely, $u=\sqrt{y_{+} y_{-}}>u_{\mathrm{o}}=\frac{1}{\sqrt{2}} z_{\mathrm{o}}$ implies that one is to integrate over a "triangle" instead of a "square" in the $\ln y_{ \pm}$plane, $\ln y_{+}+\ln y_{-}=2 \ln u>2 \ln u_{\mathrm{o}}$, and that gives an extra factor of 2 compared to (3.23). As a result, $A_{2}=\frac{1}{4}\left(\ln \frac{u_{*}}{u_{\mathrm{o}}}\right)^{2}=\frac{1}{4 \epsilon^{2}}(\ln \sqrt{\lambda})^{2}+\ldots$. We then have according to (3.34) and (3.36) $\Gamma_{1}=a_{1} A_{2}+\ldots=\frac{a_{1}}{4 \epsilon^{2}}(\ln \sqrt{\lambda})^{2}+\ldots$ which matches the result for the contribution of the one of 4 divergent factors in the amplitude (1.2) that follows from (3.39), (3.41). For higher orders one may attempt to analytically continue in $\epsilon$, effectively reversing its sign; replacing then $\frac{1}{\sqrt{\lambda}}$ by $\frac{z^{\epsilon}}{\sqrt{\lambda}}$ with $z \rightarrow \sqrt{2 y_{+} y_{-}}$under the world-sheet integral at each loop order then appears to lead to coefficients in $f^{(-2)}(\lambda)$ consistent with (3.40). It still

\footnotetext{
${ }^{40}$ That would be the case if one would simply impose a cutoff at small $z$, by, e.g., $z^{-2} \rightarrow\left(z^{2}+\varepsilon^{2}\right)^{-1}$ (cf. 9, 39). But then one would need to identify the corresponding regularization on the gauge theory side. Further complications with such a regularization include difficulties in finding the minimal surface in the presence of the regulator as well as in finding a consistent Green-Schwarz action.
} 
remains to be seen if there is a systematic version of dimensional regularization that may allow one to capture the strong-coupling expansion not only for $f(\lambda)$ but also for $g(\lambda)$.

\section{Acknowledgments}

We are grateful to L.F. Alday, Z. Bern, L. Dixon, S. Frolov, G. Korchemsky, D. Kosower, T. McLoughlin and especially J. Maldacena for useful communications and discussions. The work of M.K. was supported by the National Science Foundation under grant No. PHY-0653357. R.R. acknowledges the support of the National Science Foundation under grant PHY-0608114. A.T. was supported in part by the DOE grant DE-FG02-91ER40690. A.A.T. acknowledges the support of the PPARC, INTAS 03-51-6346, EC MRTN-CT-2004-005104 and the RS Wolfson award.

\section{Appendix A: Construction of $\mathcal{O}(\epsilon)$ correction to the 4-cusp solution}

In this Appendix we describe the construction of the $\mathcal{O}(\epsilon)$ correction to the 4-cusp Wilson loop solution (found in original $A d S_{5} \times S^{5}$ "unregularized" form in [26]) and outline the structure of the quadratic fluctuation Lagrangian.

To set up the stage, consider the general Lagrangian

$$
L_{\epsilon}=[h(\Phi)]^{\epsilon} L_{\epsilon=0}(\Phi)
$$

and let $\Phi=\Phi_{0}$ be a solution of the Lagrangian $L_{\epsilon=0}(\Phi)$. We are interested in finding the function $\Phi_{1}$ such that $\Phi_{\epsilon}=\Phi_{0}+\epsilon \Phi_{1}$ is a solution of the Lagrangian $L_{\epsilon}$ to leading and next-toleading order in the expansion in $\epsilon \rightarrow 0$. As mentioned previously, it is not a priori clear that such an expansion is sufficient for testing the conjectures of [26] and [24]; still, it gives some information on a class of functions that may appear in the complete solution.

The strategy is relatively straightforward. First, we expand $L_{\epsilon=0}$ around $\Phi_{0}$. The inclusion of the additional factor $h^{\epsilon}$ modifies the equations of motion of fluctuations by potential terms. The solution to these deformed equations yield the desired correction.

Indeed, the expansion of $L_{\epsilon=0}(\Phi)$ around the solution $\Phi=\Phi_{0}$ has the following structure:

$$
L_{\epsilon=0}\left(\Phi_{0}+\epsilon \phi\right)=L_{\epsilon=0}\left(\Phi_{0}\right)+\epsilon \partial_{a}\left(c^{a} \phi\right)+\epsilon^{2} \phi \cdot K\left(\Phi_{0}\right) \cdot \phi+\epsilon^{2} \partial_{a}\left(\phi \cdot V^{a}\left(\Phi_{0}\right) \cdot \phi\right)+\mathcal{O}\left(\epsilon^{3}\right)
$$

The first term here is simply the value of the Lagrangian on the classical solution. The second and fourth terms are total derivatives that integrate to zero at the level of the action. $K\left(\Phi_{0}\right)$ is the kinetic operator of the small fluctuations around $\Phi_{0}$ and, in general, it may be a function of the world sheet coordinates.

With this starting point it is easy to understand the structure of the equations determining $\Phi_{1}$. Indeed, in the presence of $h^{\epsilon}$ the field configuration $\Phi_{0}$ is no longer a solution of the 
equations of motion. Instead, at the level of the action, the fluctuations $\phi$ exhibit a tadpole which, to leading order in $\epsilon$ is just

$$
L_{\text {tadpole }}=-\epsilon \phi c^{a} \partial_{a} \ln h\left(\Phi_{0}\right)
$$

Thus, to summarize, the equations determining $\Phi_{1}$ are the equations of motion of the fluctuations around $\Phi_{0}$ at $\epsilon=0$ deformed by a potential generated by the tadpole Lagrangian (A.3).

In our case we have $h(\Phi) \equiv 1 / z$ and the undeformed solution $\Phi_{0}$ is the 4 -cusp solution of [26] in the conformal gauge

$$
\begin{aligned}
z_{0} & =\frac{a}{\cosh u_{2} \cosh u_{1}+b \sinh u_{2} \sinh u_{1}} \\
y_{00} & =\frac{a \sqrt{1+b^{2}} \sinh u_{2} \sinh u_{1}}{\cosh u_{2} \cosh u_{1}+b \sinh u_{2} \sinh u_{1}} \\
y_{01} & =\frac{a \sinh u_{2} \cosh u_{1}}{\cosh u_{2} \cosh u_{1}+b \sinh u_{2} \sinh u_{1}} \\
y_{02} & =\frac{a \cosh u_{2} \sinh u_{1}}{\cosh u_{2} \cosh u_{1}+b \sinh u_{2} \sinh u_{1}} \\
y_{03} & =0
\end{aligned}
$$

where the parameters $a$ and $b$ are related to the Mandelstam invariants [26] and $\left(u_{1}, u_{2}\right)=(\tau, \sigma)$.

The relation between the 1-cusp and 4-cusp solutions implies that a convenient choice of the basis of fluctuation fields

$$
z=z_{0}+\epsilon \delta z, \quad y_{i}=y_{0 i}+\epsilon \delta y_{i}
$$

is given by $\left(\tilde{y}, \tilde{\varphi}_{1}, \tilde{\varphi}_{2}, \tilde{\phi}, \tilde{x}\right)$

$$
\begin{aligned}
\delta z= & \frac{a}{2\left(\cosh u_{2} \cosh u_{1}+b \sinh u_{2} \sinh u_{1}\right)^{2}}\left[-2 \sqrt{1+b^{2}} \tilde{y}\right. \\
& -\left(\cosh u_{1} \sinh u_{2}+b \cosh u_{2} \sinh u_{1}\right)\left(\tilde{\varphi}_{1}+\tilde{\varphi}_{2}\right) \\
& -\left(\cosh u_{2} \sinh u_{1}+b \cosh u_{1} \sinh u_{2}\right)\left(\tilde{\varphi}_{1}-\tilde{\varphi}_{2}\right) \\
& \left.+2\left(b \cosh u_{1} \cosh u_{2}+\sinh u_{1} \sinh u_{2}\right) \tilde{\phi}\right] \\
\delta y_{0}= & \frac{a}{4\left(\cosh u_{2} \cosh u_{1}+b \sinh u_{2} \sinh u_{1}\right)^{2}}\left[4\left(b \cosh u_{1} \cosh u_{2}-\sinh u_{1} \sinh u_{2}\right) \tilde{y}\right. \\
& +\sinh \left(2 u_{1}\right)\left(\tilde{\varphi}_{1}+\tilde{\varphi}_{2}\right)+\sinh \left(2 u_{2}\right)\left(\tilde{\varphi}_{1}-\tilde{\varphi}_{2}\right) \\
& \left.-2 \sqrt{1+b^{2}}\left(\cosh \left(2 u_{1}\right)+\cosh \left(2 u_{2}\right)\right) \tilde{\phi}\right] \\
\delta y_{1}= & \frac{a}{2\left(\cosh u_{2} \cosh u_{1}+b \sinh u_{2} \sinh u_{1}\right)^{2}}\left[-2 \sqrt{1+b^{2}} \cosh u_{1} \sinh u_{2} \tilde{y}\right. \\
& +\cosh u_{1}\left(\tilde{\varphi}_{1}+\tilde{\varphi}_{2}\right)-b \sinh u_{2}\left(\tilde{\varphi}_{1}-\tilde{\varphi}_{2}\right) \\
& \left.-\left(\sinh ^{2}\left(2 u_{1}\right)-b \sinh \left(2 u_{2}\right)\right) \tilde{\phi}\right] \\
\delta y_{2}= & \frac{a}{2\left(\cosh _{2} u_{2} \cosh u_{1}+b \sinh u_{2} \sinh _{1} u_{1}^{2}\right.}\left[-2 \sqrt{1+b^{2}} \cosh u_{2} \sinh u_{1} \tilde{y}\right. \\
& +\cosh ^{2} u_{2}\left(\tilde{\varphi}_{1}+\tilde{\varphi}_{2}\right)-b \sinh ^{2} u_{1}\left(\tilde{\varphi}_{1}-\tilde{\varphi}_{2}\right)
\end{aligned}
$$




$$
\delta y_{3}=\frac{\left.-\left(\sinh \left(2 u_{2}\right)-b \sinh \left(2 u_{1}\right)\right) \tilde{\phi}\right]}{\cosh u_{2} \cosh u_{1}+b \sinh u_{2} \sinh u_{1}}
$$

This choice leads to constant coefficients in the operator $K_{0}$.

It is straightforward (though somewhat tedious) to find the equations corrected by the presence of the regulator. Only the solution obeying the Virasoro constraints is physically interesting. Since for $\epsilon=0$ the solution does obeys the constraints, the resulting conditions on the correction are not sensitive to the regulator. Indeed, it turns out that the vanishing of the $2 \mathrm{~d}$ stress tensor of the fluctuation fields requires that $\left(\tilde{y}, \tilde{\varphi}_{1}, \tilde{\varphi}_{2}, \tilde{\phi}, \tilde{x}\right)$ are related by

$$
\begin{aligned}
& \left(\partial_{u_{1}}-\partial_{u_{2}}\right) \tilde{\varphi}_{1}-\left(\partial_{u_{1}}+\partial_{u_{2}}\right) \tilde{\varphi}_{2}=0 \\
& \left(\partial_{u_{1}}+\partial_{u_{2}}\right) \tilde{\varphi}_{1}+\left(\partial_{u_{1}}-\partial_{u_{2}}\right) \tilde{\varphi}_{2}=4 \tilde{\phi} .
\end{aligned}
$$

The solution of these equations can be parametrized by a single arbitrary function $G$ :

$$
\tilde{\varphi}_{1}=\left(\partial_{u_{1}}+\partial_{u_{2}}\right) G, \quad \tilde{\varphi}_{2}=\left(\partial_{u_{1}}-\partial_{u_{2}}\right) G, \quad\left(\partial_{u_{1}}^{2}+\partial_{u_{2}}^{2}\right) G=2 \tilde{\phi}
$$

Further using the Virasoro constraints to simplify the remaining equations of motion it is not hard to see that they in fact decouple. The remaining functions $(\tilde{x}, \tilde{y}, \tilde{\phi})$ are determined by

$$
\begin{aligned}
& \left(\partial_{u_{1}}^{2}+\partial_{u_{2}}^{2}-4\right) \tilde{\phi}-\frac{\sinh u_{1} \sinh u_{2}+b \cosh u_{1} \cosh u_{2}}{\cosh u_{1} \cosh u_{2}+b \sinh u_{1} \sinh u_{2}}=0 \\
& \left(\partial_{u_{1}}^{2}+\partial_{u_{2}}^{2}-2\right) \tilde{x}=0, \quad \sqrt{1+b^{2}} \\
& \left(\partial_{u_{1}}^{2}+\partial_{u_{2}}^{2}-2\right) \tilde{y}-\frac{\sinh u_{1} \sinh u_{2}}{\cosh u_{1} \cosh u_{2}+b}=0
\end{aligned}
$$

The solution for $\tilde{\phi}$ is then the source for the equation (A.8) determining $G$.

The $\epsilon=0$ solution already obeys the boundary conditions that relate its shape to the Mandelstam invariants of the scattering process. The corrections to the solution must therefore leave these boundary conditions unchanged. For the particular case of $s=t$ (i.e. $b=0$ ) this translates into

$$
\begin{array}{lll}
u_{2} \rightarrow \pm \infty: & \pm 2 \tilde{\phi}=\tilde{\varphi}_{1}+\tilde{\varphi}_{2}, & \tilde{y}=0 \\
u_{1} \rightarrow \pm \infty: & \pm 2 \tilde{\phi}=\tilde{\varphi}_{1}-\tilde{\varphi}_{2}, & \tilde{y}=0
\end{array}
$$

Once a solution to (A.8), (A.9) and $(\mathrm{A.10}$ ) is found, it is then straightforward to find the value of the classical action. Since the solution correctly captures only the $\mathcal{O}(\epsilon)$ deformation, the expansion of the classical action should be truncated to this order. The result reads

$$
L_{\epsilon}\left(\Phi_{\epsilon}\right)=\frac{1}{z_{0}^{\epsilon}}\left[2+4 \epsilon \partial_{u_{1}} \partial_{u_{2}} G+\mathcal{O}\left(\epsilon^{2}\right)\right]
$$

Note that $\frac{1}{\left(z_{0}+\epsilon \delta z\right)^{\epsilon}}=\frac{1}{z_{0}^{\epsilon}}+\mathcal{O}\left(\epsilon^{2}\right)$ so the regulator does not contribute to this order except for the overall factor. 
With the $\mathcal{O}(\epsilon)$ correction to the classical solution at hand, one may now proceed to compute the quadratic fluctuation Lagrangian. Only the terms of the order $\epsilon^{0}$ and $\epsilon^{1}$ are reliable.

The idea is to use as much as possible the fact that the expansion of $L_{\epsilon=0}$ around the solution (A.4) has - up to total derivatives - constant coefficients if the fluctuation fields are chosen similarly to eq.(A.6). With this in mind, the structure of the quadratic fluctuation Lagrangian contains three types of terms:

1) the quadratic fluctuation part of $L_{\epsilon=0}$ expanded around the $\epsilon=0$ solution

2) the cubic vertices of $L_{\epsilon=0}$ expanded around the $\epsilon=0$ solution in which one of the fields is replaced by the $\mathcal{O}(\epsilon)$ correction constructed above

3) the derivative of $L_{\epsilon}$ with respect to $\epsilon$ evaluated on the undeformed solution and expanded to quadratic order in fluctuations around it.

The terms of the first type have constant coefficients; the coordinate dependence in the terms of the second type arises entirely from from the $\mathcal{O}(\epsilon)$ correction to the solution. The position dependence in third type of terms arises from the regulator as well as from the total derivative terms that integrate to zero in the absence of the regulator.

The calculation of the determinant of this operator is non-trivial due to a complicated position dependence. An expansion in $\epsilon$ is, however, possible.

Still, it is important to stress that it is unclear whether such an expansion yields the expected result for the poles in $\epsilon$. First, the leading order result has the same unfortunate features discussed in section 3 - namely, that it seems not to be regularized by non-zero $\epsilon$. Also, the orders of poles in $\epsilon$ appearing at higher orders strongly depend on the large distance structure of the correction to the $\epsilon=0$ solution. It is possible that the higher order corrections bring increasingly higher order poles and the expected pole structure of the IR singular part of the amplitude [24] arises only after all these poles are resummed.

To conclude, it appears that a solution exact to all orders in $\epsilon$ is required for such an approach to have a chance of leading to a finite contribution to the expectation value of the 4-cusp Wilson loop.

\section{Appendix B: An attempt of modified dimensional regularization prescription}

We have seen in section 3 that a naive application of the regularization scheme suggested in [26] to the single-cusp momentum space Wilson line does not appear to reproduce the structure of the gauge theory Sudakov form-factor $A_{d i v} \equiv\left(\mathcal{M}^{[g g \rightarrow 1]}\right)^{1 / 2}$ beyond the leading strong-coupling order. It turns out, however, that it is possible to suggest a formal prescription which accomplishes this for the leading $\frac{1}{\epsilon^{2}}$ pole and, simultaneously, reproduces the finite part of the logarithm of the exponentiated 4-gluon scattering amplitude [24].

Let us consider applying the dimensional regularization of [26] not at the level of the classical superstring action but only to the divergent quantities that arise during the calculation. More precisely, using the $\epsilon=0$ solution for the minimal surface and taking into account its homogeneity property, we can first factorize its area as was done in section 2.2 and then regularize 
the area using the induced metric containing the $\frac{1}{z^{\epsilon}}$ factor. Demanding that the coefficient of the second order pole in $\epsilon$ in the expectation value of the single-cusp Wilson line matches that in the Sudakov form factor will relate the dimensional continuation parameters on the gauge and the string sides. Applying this relation to the 4-cusp Wilson loop we may then reproduce the conjectured exponential structure of the amplitude [23, 24] to all orders in string perturbation theory.

As discussed in section 2, the homogeneity of the single-cusp solution and its relation to the spinning closed string solution imply that the expectation value of the Wilson loop factor satisfies

$$
\ln \langle W\rangle=\ln \mathcal{Z}=-f(\lambda) A_{2} .
$$

In general, $W$ may stand for all Wilson loops whose corresponding minimal surfaces are related by symmetries of $A d S_{5} \times S^{5}$ to the single-cusp Wilson loop surface. The difference between the minimal surfaces will manifest itself in the values of their regularized areas $A_{2}$.

An interesting observation is that while the expression above does not immediately reproduce the singular part of the 4-gluon scattering amplitude, it does, however, correctly capture its finite part. Indeed, the calculation of the regularized area in [26] yields

$$
\begin{aligned}
A_{2}^{4-\text { cusp }} & =2 A_{\text {div }, s}^{\text {string }}+2 A_{\text {div }, t}^{\text {string }}-\frac{1}{8}\left(\ln \frac{s}{t}\right)^{2}+\text { const }, \\
A_{\text {div }, s}^{\text {string }} & =\left[\frac{1}{2 \epsilon_{\text {string }}^{2}}+\frac{1-\ln 2}{4 \epsilon_{\text {string }}}\right]\left(\frac{\mu^{2}}{|s|}\right)^{\frac{1}{2} \epsilon_{\text {string }}} .
\end{aligned}
$$

Using it in equation (B.1) implies that the terms unrelated to the IR singularities are

$$
\widetilde{\mathcal{F}}_{1}=\frac{f(\lambda)}{8}\left(\ln \frac{s}{t}\right)^{2}+\text { const }
$$

which reproduces (up to a constant shift) the expected structure of the finite part of the 4-gluon amplitude [24, 26, 25].

Assuming that there should indeed be a relation between the null momentum Wilson loops and the gluon amplitudes, the observation above suggests that we should aim at identifying $f(\lambda) A_{\mathrm{div}, s}^{\text {string }}$ with the logarithm of the Sudakov form factor, namely

$$
f(\lambda) A_{\text {div }, s}\left(\epsilon_{\text {string }}, \mu_{\text {string }}\right)=\frac{1}{8 \epsilon_{\text {gauge }}^{2}} f^{(-2)}(\tilde{\lambda})+\frac{1}{4 \epsilon_{\text {gauge }}} g^{(-1)}(\tilde{\lambda}), \quad \tilde{\lambda} \equiv \lambda\left(\frac{\mu_{\text {gauge }}^{2}}{|s|}\right)^{\epsilon_{\text {gauge }}}
$$

This identification should hold up to $\mathcal{O}\left(\epsilon_{\text {string }}\right)$ corrections. By comparing the second order poles it is easy to see that they can be made to agree if we choose 41

$$
\epsilon_{\text {string }}=\epsilon_{\text {gauge }} \sqrt{\frac{f(\lambda)}{f^{(-2)}(\lambda)}}
$$

\footnotetext{
${ }^{41}$ This identification also leads to a successful comparison of the coefficient of the double-pole in the vev of the single-cusp Wilson line and that of the Sudakov form factor. Indeed, the equation (3.37) implies that

$$
-\ln \left\langle W_{1 \text { cusp }}\right\rangle=\frac{f(\lambda)}{\epsilon_{\text {string }}^{2}}+\mathcal{O}\left(\frac{1}{\epsilon_{\text {string }}}\right)=\frac{f^{(-2)}(\lambda)}{\epsilon_{\text {gauge }}^{2}}+\mathcal{O}\left(\frac{1}{\epsilon_{\text {gauge }}}\right) .
$$
}


It appears hard if not impossible, however, to match the simple pole and the finite parts on the l.h.s. and r.h.s. of the equation (B.5). To match the simple pole it seems necessary to demand that $\left(\mu^{2} / s\right)_{\text {string }}=\left(\mu^{2} / s\right)_{\text {gauge }}^{q(\lambda)}$. Further ad hoc adjustments appear to be needed in order to match the finite parts of (B.5).

\section{References}

[1] D.J. Gross and F. Wilczek, "Asymptotically free gauge theories. 2," Phys. Rev. D 9, 980 (1974). H. Georgi and H. D. Politzer, "Electroproduction scaling in an asymptotically free theory of strong interactions," Phys. Rev. D 9, 416 (1974).

[2] E. G. Floratos, D. A. Ross and C. T. Sachrajda, "Higher Order Effects In Asymptotically Free Gauge Theories. 2. Flavor Singlet Wilson Operators And Coefficient Functions," Nucl. Phys. B 152, 493 (1979). A. Gonzalez-Arroyo and C. Lopez, "Second Order Contributions To The Structure Functions In Deep Inelastic Scattering. 3. The Singlet Case," Nucl. Phys. B 166, 429 (1980). A. V. Kotikov, L. N. Lipatov, A. I. Onishchenko and V. N. Velizhanin, "Three-loop universal anomalous dimension of the Wilson operators in N = 4 SUSY Yang-Mills model," Phys. Lett. B 595, 521 (2004) [Erratum-ibid. B 632, 754 (2006)] arXiv:hep-th/0404092.

[3] A. M. Polyakov, "Gauge Fields As Rings Of Glue," Nucl. Phys. B 164, 171 (1980). R. A. Brandt, F. Neri and M. A. Sato, "Renormalization Of Loop Functions For All Loops," Phys. Rev. D 24, 879 (1981).

[4] N. S. Craigie and H. Dorn, "On The Renormalization And Short Distance Properties Of Hadronic Operators In QCD," Nucl. Phys. B 185, 204 (1981). G. P. Korchemsky and A. V. Radyushkin, "Renormalization of the Wilson Loops Beyond the Leading Order," Nucl. Phys. B 283, 342 (1987). I. I. Balitsky and V. M. Braun, "Evolution Equations for QCD String Operators," Nucl. Phys. B 311, 541 (1989).

[5] G. P. Korchemsky, "Asymptotics of the Altarelli-Parisi-Lipatov Evolution Kernels of Parton Distributions," Mod. Phys. Lett. A 4, 1257 (1989). G. P. Korchemsky and G. Marchesini, "Structure function for large $\mathrm{x}$ and renormalization of Wilson loop," Nucl. Phys. B 406, 225 (1993) arXiv:hep-ph/9210281. A. Bassetto, I. A. Korchemskaya, G. P. Korchemsky and G. Nardelli, "Gauge invariance and anomalous dimensions of a light cone Wilson loop in lightlike axial gauge," Nucl. Phys. B 408, 62 (1993) arXiv:hep-ph/9303314].

[6] Y. Makeenko, P. Olesen and G. W. Semenoff, "Cusped SYM Wilson loop at two loops and beyond," Nucl. Phys. B 748, 170 (2006) [arXiv:hep-th/0602100].

[7] S. S. Gubser, I. R. Klebanov and A. M. Polyakov, "A semi-classical limit of the gauge/string correspondence," Nucl. Phys. B 636, 99 (2002) arXiv:hep-th/0204051].

[8] M. Kruczenski, "A note on twist two operators in $\mathrm{N}=4$ SYM and Wilson loops in Minkowski signature," JHEP 0212, 024 (2002) arXiv:hep-th/0210115]. 
[9] Y. Makeenko, "Light-cone Wilson loops and the string / gauge correspondence," JHEP 0301, 007 (2003) [arXiv:hep-th/0210256].

[10] S. Frolov and A. A. Tseytlin, "Semiclassical quantization of rotating superstring in AdS(5) x S(5)," JHEP 0206, 007 (2002) arXiv:hep-th/0204226].

[11] S. Frolov, A. Tirziu and A. A. Tseytlin, "Logarithmic corrections to higher twist scaling at strong coupling from AdS/CFT," Nucl. Phys. B 766, 232 (2007) [arXiv:hep-th/0611269].

[12] R. Roiban, A. Tirziu and A. A. Tseytlin, "Two-loop world-sheet corrections in AdS(5) x S5 superstring," arXiv:0704.3638 [hep-th].

[13] J. M. Maldacena, "Wilson loops in large N field theories," Phys. Rev. Lett. 80, 4859 (1998) arXiv:hep-th/9803002]. S. J. Rey and J. T. Yee, "Macroscopic strings as heavy quarks in large N gauge theory and anti-de Sitter supergravity," Eur. Phys. J. C 22, 379 (2001) arXiv:hep-th/9803001.

[14] N. Drukker, D. J. Gross and H. Ooguri, "Wilson loops and minimal surfaces," Phys. Rev. D 60, 125006 (1999) arXiv:hep-th/9904191.

[15] R. Kallosh and A. A. Tseytlin, "Simplifying superstring action on AdS(5) x S(5)," JHEP 9810, 016 (1998) arXiv:hep-th/9808088].

[16] S. Forste, D. Ghoshal and S. Theisen, "Stringy corrections to the Wilson loop in N $=4$ super Yang-Mills theory," JHEP 9908, 013 (1999) [arXiv:hep-th/9903042].

[17] N. Drukker, D. J. Gross and A. A. Tseytlin, "Green-Schwarz string in AdS(5) x S(5): Semiclassical partition function," JHEP 0004, 021 (2000) arXiv:hep-th/0001204.

[18] S. Frolov and A. A. Tseytlin, "Multi-spin string solutions in AdS(5) x S5," Nucl. Phys. B 668, 77 (2003) arXiv:hep-th/0304255.

[19] G. Arutyunov, J. Russo and A. A. Tseytlin, "Spinning strings in AdS(5) x S**5: New integrable system relations," Phys. Rev. D 69, 086009 (2004) arXiv:hep-th/0311004.

[20] G. P. Korchemsky and A. V. Radyushkin, "Loop Space Formalism And Renormalization Group For The Infrared Asymptotics Of QCD," Phys. Lett. B 171, 459 (1986). G. P. Korchemsky, "On Near Forward High-Energy Scattering In QCD," Phys. Lett. B 325, 459 (1994) arXiv:hep-ph/9311294]. I. A. Korchemskaya and G. P. Korchemsky, "High-energy scattering in QCD and cross singularities of Wilson loops," Nucl. Phys. B 437, 127 (1995) arXiv:hep-ph/9409446].

[21] A. Sen, "Asymptotic Behavior Of The Sudakov Form-Factor In QCD," Phys. Rev. D 24, 3281 (1981). G. P. Korchemsky, "Double logarithmic asymptotics in QCD" Phys. Lett. B 217, 330 (1989).

[22] L. Magnea and G. Sterman, "Analytic continuation of the Sudakov form-factor in QCD," Phys. Rev. D 42, 4222 (1990). G. Sterman and M. E. Tejeda-Yeomans, "Multi-loop amplitudes and resummation," Phys. Lett. B 552, 48 (2003) [arXiv:hep-ph/0210130]. 
[23] C. Anastasiou, Z. Bern, L. J. Dixon and D. A. Kosower, Phys. Rev. Lett. 91, 251602 (2003) arXiv:hep-th/0309040].

[24] Z. Bern, L. J. Dixon and V. A. Smirnov, "Iteration of planar amplitudes in maximally supersymmetric Yang-Mills theory at three loops and beyond," Phys. Rev. D 72, 085001 (2005) arXiv:hep-th/0505205|.

[25] J. M. Drummond, G. P. Korchemsky and E. Sokatchev, "Conformal properties of fourgluon planar amplitudes and Wilson loops," arXiv:0707.0243 [hep-th].

[26] L. F. Alday and J. Maldacena, "Gluon scattering amplitudes at strong coupling," arXiv:0705.0303 [hep-th].

[27] D. J. Gross and P. F. Mende, "The High-Energy Behavior of String Scattering Amplitudes," Phys. Lett. B 197, 129 (1987).

[28] J. Dai, R. G. Leigh and J. Polchinski, "New Connections Between String Theories," Mod. Phys. Lett. A 4, 2073 (1989).

[29] A. A. Migdal, "Momentum Loop Dynamics And Random Surfaces In QCD," Nucl. Phys. B 265, 594 (1986).

[30] A. M. Polyakov, "String theory and quark confinement," Nucl. Phys. Proc. Suppl. 68, 1 (1998) arXiv:hep-th/9711002.

[31] R. R. Metsaev and A. A. Tseytlin, "Type IIB superstring action in AdS(5) x S(5) background," Nucl. Phys. B 533, 109 (1998) arXiv:hep-th/9805028.

[32] A. A. Tseytlin and K. Zarembo, "Wilson loops in N = 4 SYM theory: Rotation in S(5)," Phys. Rev. D 66, 125010 (2002) [arXiv:hep-th/0207241].

[33] M. Kruczenski, "Spiky strings and single trace operators in gauge theories," JHEP 0508, 014 (2005) arXiv:hep-th/0410226]. M. Kruczenski, J. Russo and A. A. Tseytlin, "Spiky strings and giant magnons on S5," JHEP 0610, 002 (2006) [arXiv:hep-th/0607044]. R. Ishizeki and M. Kruczenski, "Single spike solutions for strings on S2 and S3," arXiv:0705.2429 [hep-th].

[34] R. Kallosh and J. Rahmfeld, "The GS string action on AdS(5) x S(5)," Phys. Lett. B 443, 143 (1998) arXiv:hep-th/9808038.

[35] R. R. Metsaev and A. A. Tseytlin, "Superstring action in AdS(5) x S(5): kappa-symmetry light cone gauge," Phys. Rev. D 63, 046002 (2001) [arXiv:hep-th/0007036].

[36] G. W. Gibbons, M. B. Green and M. J. Perry, "Instantons and Seven-Branes in Type IIB Superstring Theory," Phys. Lett. B 370, 37 (1996) arXiv:hep-th/9511080].

[37] M. T. Grisaru, P. S. Howe, L. Mezincescu, B. Nilsson and P. K. Townsend, "N=2 Superstrings In A Supergravity Background," Phys. Lett. B 162, 116 (1985). 
[38] E.I. Buchbinder, "Infrared limit of gluon amplitudes at strong coupling", arXiv:0706.2015 [hep-th].

[39] S. Abel, S. Forste and V. V. Khoze, "Scattering amplitudes in strongly coupled N=4 SYM from semiclassical strings in AdS," arXiv:0705.2113 [hep-th].

[40] A. Brandhuber, P. Heslop and G. Travaglini, "MHV Amplitudes in N=4 Super Yang-Mills and Wilson Loops," arXiv:0707.1153 [hep-th]. 